\title{
La production d'énergie électrique par éoliennes
}

\author{
PAR I. VADOT \\ INGÉNIEUR-CONSEIL AUX F́TABLISSEMENTS NEYRPIC
}

English text, p. 526

\begin{abstract}
Deux problèmes fondamentaux pour l'étude des aérogénérateurs sont axaminés dans cet article: la détermination de la dimension de l'appareil et celle de la puissance équipée. Des considérations économiques et technologiques permettent de défnir les caractéristiques de l'appareil le plus abantageux. Il apparaît que, compte tenu des disponibilités en énergie eolienne dans la zone atlantique, l'utilisation d'aérogénérateurs est dès maintenant intéressante.
\end{abstract}

Deux faits essentiels dominent le problème de l'utilisation de l'énergie du vent : d'une part la grande dilution de cette énergie dans l'espace, d'autre part son irrégularité à la fois considérable et imprévisible.

Nous nous trouvons là en présence d'une de ces formes d'énergie dont l'apparente et séduisante gratuité donne toute son importance à l'économie de la construction. Quelques chiffres fixeront les idées.

Dans un vent de $10 \mathrm{~m} / \mathrm{s}$, la puissance maximum qu'il est possible de recueillir, avec une machine parfaite, est de l'ordre de $0,3 \mathrm{~kW}$ par m". Pratiquement, d'ailleurs, les imperfections des appareils ne permettent d'obtenir que $0,2 \mathrm{~kW}$ par m². Il n'existe aucun site où l'on dispose d'une telle puissance de façon régulière, car la vitesse du vent varie sans cesse; d'une valeur nulle, pendant les périodes de calme, elle peut atteindre au cours des tempêtes $50 \mathrm{~m} / \mathrm{s}$ et même plus, ce qui conduirait à une puissance par $m^{2} 125$ fois plus grande que celle envisagée ici, soit, dans nos hypothèses, $25 \mathrm{~kW}$ par $\mathrm{m}^{2}$.

L'irrégularité dans le temps est telle que le nombre d'heures pendant lequel on dispose d'une certaine puissance diminue rapidement quand cette puissance augmente, en sorte qu'il est tout à fait hors de question de construire un appareil capable de fournir $25 \mathrm{~kW}$ par $\mathrm{m}^{2}$. Des considéra- tions économiques amèneront à choisir un chiffre beaucoup plus bas, qui se situera aux alentours de 0,2 ou $0,3 \mathrm{~kW}$ par $\mathrm{m}^{2}$ pour un site moyennement venté. L'appareil devra néanmoins être capable de résister aux vents les plus violents.

Le premier souci sera de mettre au point une construction qui reste économique malgré l'écart énorme existant entre la puissance moyennement récupérable et la puissance accidentelle maximum qui traverse la machine. Nous avons indiqué au cours d'une précédente étude (Etude synoptique des différents types d'éoliennes, la Houille Blanche, mars-avril 1957) quelles étaient les principales dispositions qui permettaient de supporter les grandes variations de vitesse de vent. La nécessité de s'accommoder de l'irrégularité, dans le temps, de la production d'énergie est une autre source de préoccupation.

On a cherché à maintes reprises à remédier à l'irrégularité de l'énergie du vent en l'accumulant pour la restituer au moment convenable et rendre la consommation indépendante de la production. Presque tous les moyens connus ont été employés ou étudiés : accumulation hydraulique par pompage et turbinage, accumulation cinétique par volant à grande vitesse, accumulation thermique par production directe de chaleur au moyen de frein genre Froude, accumulation élec- 
trique par batterie, accumulation chimique par l'intermédiaire de l'électrolyse avec production d'hydrogène et d'oxygène, etc... L'accumulation est une formule, la compensation en est une autre; elle consiste à faire travailler la machine éolienne en parallèle avec une autre source d'énergie, dont la production sera, vis-à-vis de la consommation, complémentaire de la production éolienne. Une troisième solution enfin n'est autre que le choix d'un type de consommation qui puisse s'adapter sans inconvénient aux irrégularités de la production; c'était en somme le cas de la mouture du blé à l'époque où les moulins à vent méritaient leur nom.
Mis à part certains cas très particuliers, l'irrégularité de l'énergie du vent conduit à deux applications importantes : le pompage de l'eau et la production d'énergie électrique. La question du pompage ayant été examinée dans une précédente étude (Le pompage de l'eau par éoliennes, la Houille Blanche, septembre 1957), nous nous attacherons maintenant à exposer les différents problèmes soulevés par la construction des machines éoliennes, en vue de la production d'énergie électrique.

Certes, les conditions d'exploitation d'un aérogénérateur peuvent être très diverses, mais pour la simplicité de l'exposé, il sera commode de

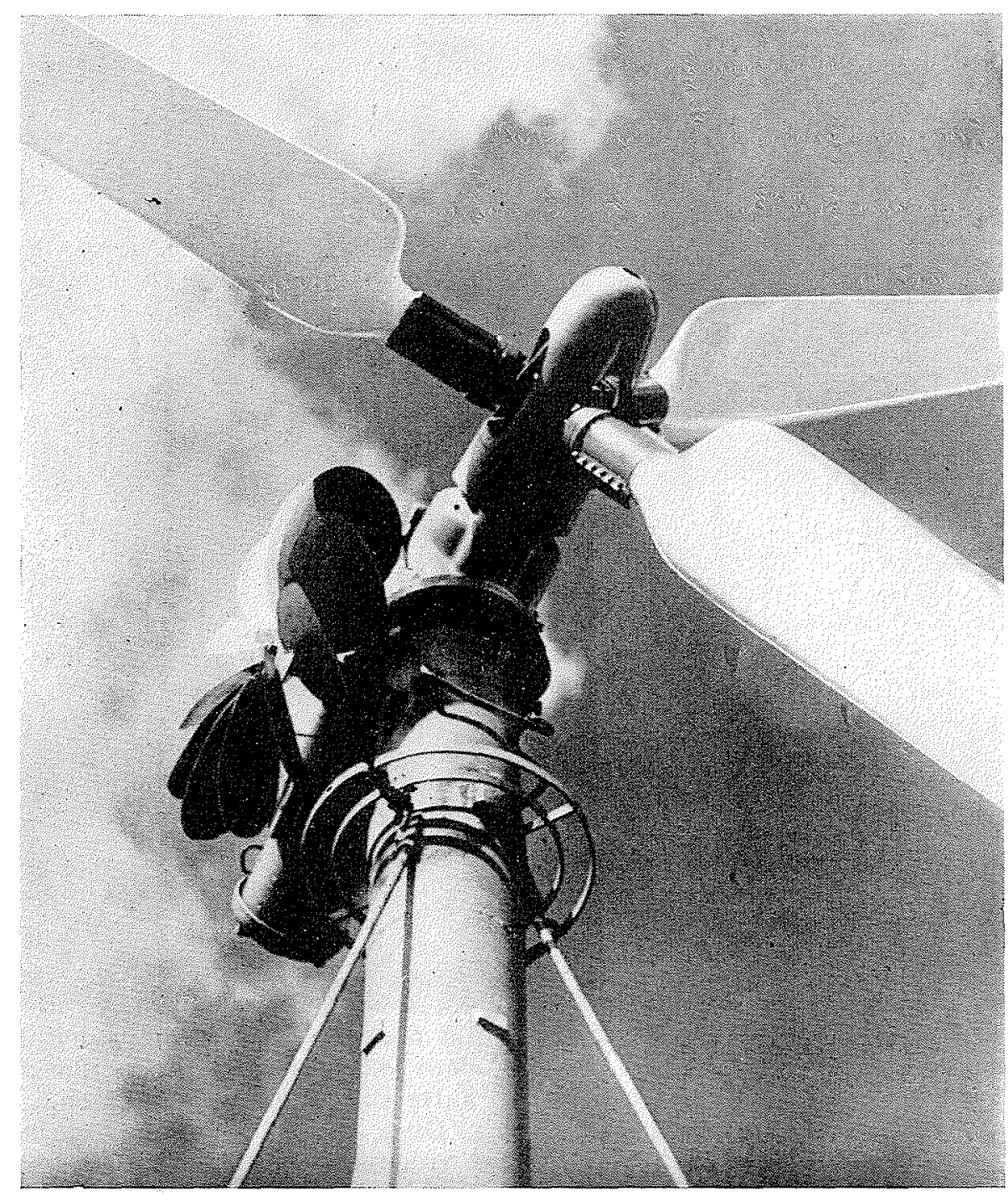

Aérogénérateur Allgaïer, système Hütter, de petite puissance, diamètre $10 \mathrm{~m}$, pouvant être équipé soit de génératrice de courant continu, soit de génératrice asynchrone, de 6 à $8 \mathrm{~kW}$ suivant les vents. La conception mécanique est nettement influencée par le souci de réalisation en série.

Allgaier small-power aerogenerator, of $10 \mathrm{~m}$ diameter, designed by Hütter. It can be equipped either with a direct-current generator or an asynchronous generator of 6 to $8 \mathrm{~kW}$, according to the winds. The mechanical design is clearly influenced by the prospect of mass production. 
considérer deux modes d'exploitation. Nous envisagerons ainsi, d'une part, le cas où l'aérogénérateur est appelé à fonctionner isolément, d'autre part, le cas où il fonctionne en parallèle avec une autre source d'énergie électrique. Celte autre source étant supposée d'une puissance suffisante pour couvrir, le cas échéant, tous les besoins de la consommation.

L'aérogénérateur isolé devra évidemment fonctionner avec accumulation. Sauf le cas où l'énergie du vent serait utilisée uniquement pour le chauffage et celui où on disposerait, à proximité, d'un moyen naturel d'accumulation hydraulique, un tel aérogénérateur sera équipé pour la production de courant continu avec batterie d'accumulateurs. Ce type de machine sera généralement d'une puissance assez faible, aussi les dimensions de l'appareil seront-elles imposées par la production demandée et non par des considérations de réalisation mécanique. Dans ce cas, enfin, les conditions économiques sont assez particulières, le prix de revient de l'énergie étant bien souvent d'une importance secondaire par rapport à la possibilité de disposer de cette énergie. Du point de vue mécanique, l'entraînement d'une génératrice de courant continu et l'appareillage nécessité par la batterie d'accumulateurs n'im- posent pas une constance rigoureuse de la vitesse de rotation, aussi peut-on se contenter d'un procédé de régulation très simple qui, pour les faibles puissances, peut être le vulgaire système de l'éclipsage de la roue dans le vent. Pour les puissances moyennes, le système des pales orientables équilibrées par ressort est très satisfaisant. Les types de roue utilisés seront naturellement à grande vitesse pour faciliter l'entraînement de la génératrice. Sur les machines sérieuses, un dispositif de sécurité devra être prévu pour éviter l'emballement en cas d'incident du côté électrique. En somme, dans ses grandes lignes, l'aérogénérateur isolé sera très voisin de l'éolienne pour entraînement de pompe centrifuge.

Tout autres seront les conditions d'établissement de l'aérogénérateur destiné à fonctionner en parallèle avec une autre source d'énergie électrique, et destiné, en particulier, à débiter sur un réseau important. Ce sont alors des considérations mécaniques et économiques qui définiront les dimensions à donner à l'appareil et qui imposeront le choix et la disposition des organes. L'importance de la puissance à réaliser par unité sera telle que de nombreux points négligeables pour de petites machines devront être examinés.

\section{I. - PROBLEMES TECHNIQUES}

\section{Relation fondamentale entre puissance- diamètre et vitesse du vent.}

Lorsqu'une veine d'air traverse une section donnée, le flux total d'énergie est représenté uniquement par le flux d'énergie cinétique; ni la pression ni la température n'interviennent, puisque ces quantités sont constantes dans une atmosphère en mouvement uniforme. Pour une veine de $1 \mathrm{~m}^{2}$ de section, à la vitesse $v \mathrm{~m} / \mathrm{s}$, la puissance totale exprimée en $\mathrm{kW}$ est :

$$
\mathrm{N}_{t}=0,000613 v^{3}
$$

pour de l'air à $15^{\circ}$ et sous $760 \mathrm{~mm}$ de mercure, c'est-à-dire de poids spécifique $1,23 \mathrm{~kg} / \mathrm{m}^{3}$.

Toute cette énergie n'est pas récupérable. Il reste, en effet, une certaine quantité d'énergie cinétique dans l'air qui a traversé la roue; cette énergie cinétique restante ne peut être nulle, puisqu'il faut évacuer l'air qui a travaillé; elle ne peut en outre être récupérée. Si $v$ est la vitesse à l'amont de la roue et $v_{2}$ la vitesse à l'aval, on montre que, pour une énergie disponible donnée, l'énergie récupérable dépend du rapport $v_{2} / v$ et passe par un maximum lorsque $v_{2} / v=1 / 3$. Dans ces conditions, l'énergie récu- pérable est égale aux $16 / 27$ de l'énergie totale. Cette propriété a été établie par Betz en 1927.

En se reportant toujours à une veine de

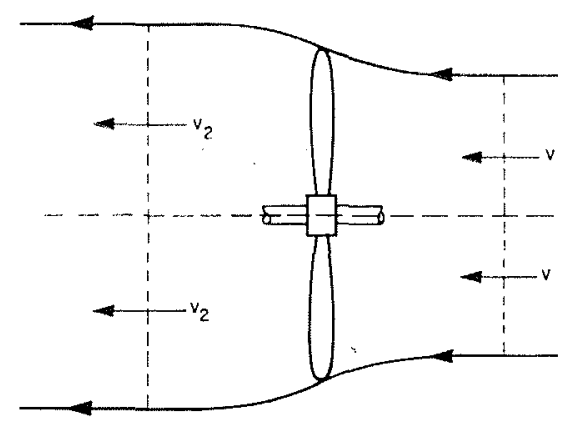

FIG. 2

$1 \mathrm{~m}^{2}$ de section, l'énergie maximum récupérable, au sens de Betz, a pour expression :

$$
\mathrm{N}_{\mathrm{I}}=(16 / 27) \times 0,000613 v^{3}
$$

soit :

$$
\mathrm{N}_{\mathrm{B}}=0,000364 v^{3}
$$

la puissance étant exprimée en $\mathrm{kW}$ et la vitesse en $\mathrm{m} / \mathrm{s}$. Une machine réelle ne récupère pas toute cette énergie; il faut encore tenir compte de ses 
différentes pertes : pertes mécaniques, pertes par frottement sur les pales, pertes par tourbillons marginaux, etc... Si $\eta$ est le rendement (par rapport à l'énergie de Betz) de la machine, la puissance effectivement récupérable sur l'arbre de la roue est :

$$
\mathrm{N}=\eta \cdot \mathrm{N}_{\mathrm{B}}=\eta \cdot(16 / 27) \mathrm{N}_{t}
$$

On a coutume de réunir en un seul terme le rendement $\eta$ et le rapport de Betz, on donne à ce terme le nom de coefficient de puissance $C_{N}$ :

$$
\mathrm{C}_{\mathrm{N}}=\frac{\mathrm{N}}{\mathrm{N}_{i}}
$$

Certains donnent à ce rapport le nom de rendement, ce qui prête à confusions, puisqu'il est établi à partir d'une puissance qui n'est pas récupérable.

Pour une roue de diamètre $D$, la puissance obtenue est :

$$
\begin{gathered}
\mathrm{N}=\frac{\pi \mathrm{D}^{2}}{4} \cdot \eta \cdot \frac{16}{27} \cdot 0,000613 v^{3} \\
=0,000286 \cdot \eta \cdot \mathrm{D}^{2} \cdot v^{3}
\end{gathered}
$$

ou, exprimée à partir du coefficient de puissance,

$$
\mathrm{N}=0,000482 \mathrm{C}_{\mathrm{N}} \mathrm{D}^{2} v^{3}
$$

la puissance étant exprimée en $\mathrm{kW}$, le diamètre en mètres et la vitesse en $\mathrm{m} / \mathrm{s}$.

Le type de roue est généralement caractérisé par sa célérité ou rapport de la vitesse périphérique $u$ à la vitesse du vent $v$. La vitesse de rotation $n$, en $\mathrm{tr} / \mathrm{mn}$, est évidemment liée à la célérité par l'expression :

$$
n=\left(\frac{u}{v}\right) \cdot \frac{60}{\pi} \cdot \frac{v}{\mathrm{D}}
$$

S'appuyant sur les formules précédentes, on a établi un diagramme donnant, en fonction de la vitesse du vent et du diamètre de la roue, la puissance récupérable sur l'arbre et la vitesse de rotation pour différentes valeurs de $u / v$, en se basant sur une bonne valeur moyenne du rendement : $\eta=70 \%$, soit $\mathrm{C}_{\mathrm{X}}=0,415$ et pour de l'air $\grave{a} 15^{\circ}$ et sous $760 \mathrm{~mm}$ de $\mathrm{Hg}$. Ce diagramme permet, avec une bonne approximation, de calculer les dimensions d'une machine pour un avantprojet.

\section{Remarque sur la comparaison éolienne- hélicoptère.}

On est parfois étonné de la faible valeur du rendement d'un aérogénérateur rapporté à l'énergie totale de la veine d'air qui le traverse. Cette faible valeur de rendement est le propre de toute machine ne pouvant récupérer l'énergie cinétique de sortie. Un exemple frappant nous sera donné par la comparaison de l'éolienne avec l'hélicoptère. Ces deux machines, en effet, l'une motrice, l'autre réceptrice, ont de nombreux points communs et une certaine similitude d'aspect de leurs rotors. Nous basant sur les caractéristiques d'un hélicoptère de construction récente, nous allons comparer le rendement de cet appareil avec celui d'un aérogénérateur de même diamètre de rotor.

L'appareil que nous considérons est l'hélicoptère Westland-Wessex qui vient d'être mis en service dans la Royal Navy et découle du classique Sikorsky S-58 par le remplacement du moteur à piston par une turbine Napier. Cet appareil répond aux caractéristiques suivantes :

Nombre de pales du rotor.......

Diamètre du rotor............. $17 \mathrm{~m}$

Vitesse de rotation............

Puissance motrice.............

Poids total...............

$221 \mathrm{tr} / \mathrm{mn}$

$1.070 \mathrm{~kW}$

Vitesse ascensionnelle maximum.. $\quad 8 \mathrm{~m} / \mathrm{s}$

Dans ces conditions, la vitesse moyenne de la veine d'air souffiée par le rotor est de $18,6 \mathrm{~m} / \mathrm{s}$. A cette vitesse correspond en bout de pale un rapport $u / v=10,7$, valeur courante pour un hélicoptère, mais qui serait assez élevée pour une éolienne pour laquuelle la grande légèreté ne s'impose pas.

Evaluons alors l'énergie totale contenue dans la veine d'air; on obtient $\mathrm{N}_{t}=910 \mathrm{~kW}$. Le rendement de l'appareil par rapport à cette énergie est $\eta=85 \%$, valeur élevée. Mais toute cette énergie est loin d'être utilisée. En effet, si on calcule l'énergie correspondant à la montée de l'appareil à sa vitesse ascensionnelle maximum, on obtient l'énergie effectivement utilisable $\mathrm{N}=456 \mathrm{~kW}$. Et le rendement vrai de l'appareil est de $\eta=456 / 1.070=43,5 \%$. Une grande partie de l'énergie du moteur se trouve dans l'énergie cinétique irrécupérable de la veine soufflée vers le bas.

Supposons maintenant que dans la même veine d'air à $18,6 \mathrm{~m} / \mathrm{s}$ on fasse tourner un rotor d'éolienne de $17 \mathrm{~m}$ de diamètre. Avec les chiffres indiqués plus haut, on peut compter sur un rendement global de $41,5 \%$, ce qui donnera une puissance de $375 \mathrm{~kW}$. Certes, il y a un écart considérable entre les $1.070 \mathrm{~kW}$ du moteur de l'hélicoptère et les $375 \mathrm{~kW}$ récupérables sur l'arbre de l'éolienne, mais les deux machines ont des rendements très semblables, respectivement 43,5 et $41,5 \%$, car dans les deux cas les pertes sont les mêmes. 


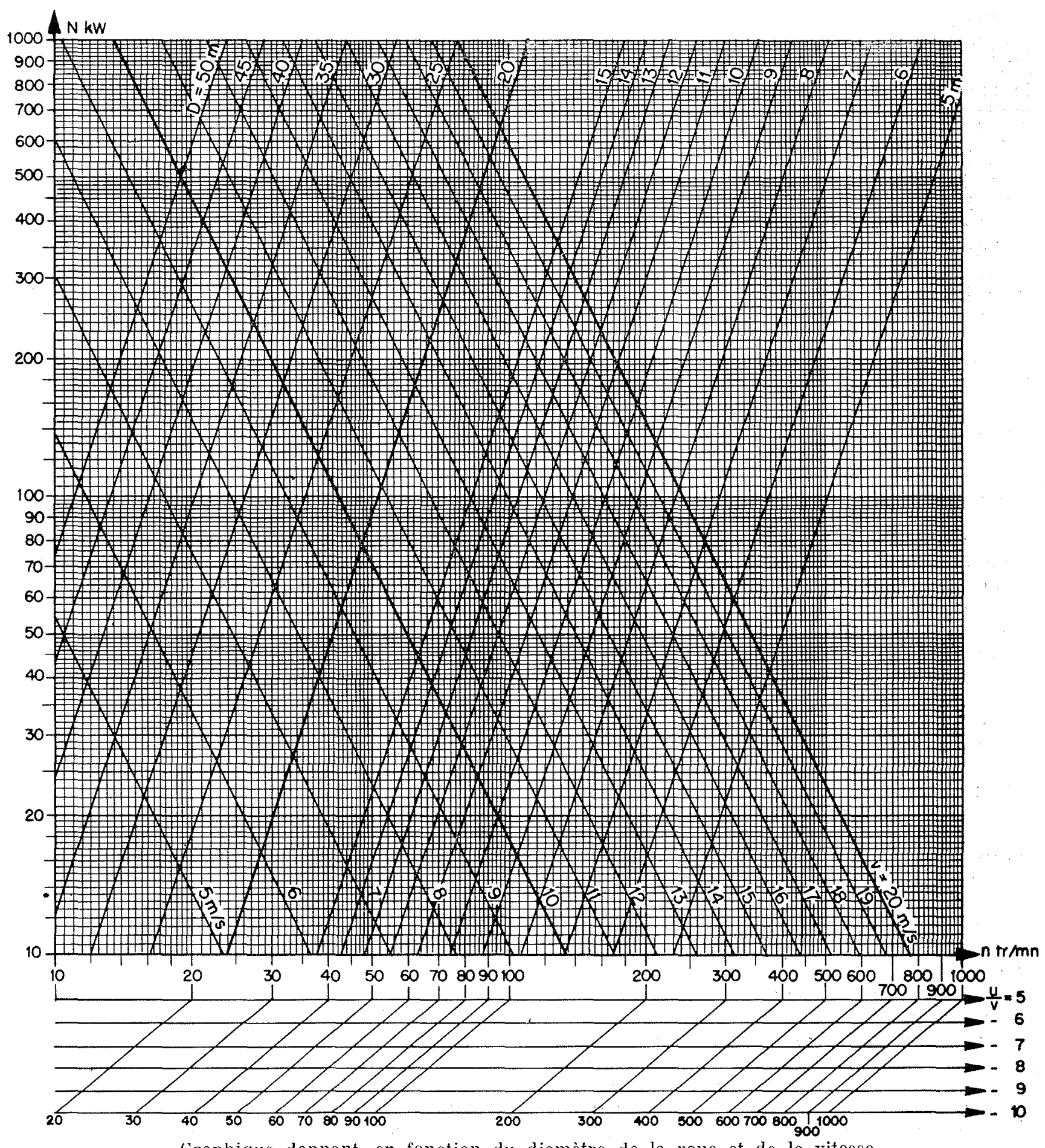

Graphique donnant, en fonction du diamètre de la roue et de la vitesse du vent, la puissance sur l'arbre et la vitesse de rotation, pour différentes valeurs de $u / v$ pour $\mathrm{C}_{\mathrm{N}}=0,415$.

Graph giving, as a function of the diameter of the rotor and of the wind speed, the power on the shaft and the speed of rotation, for different values of $\mathrm{u} / \mathrm{v}$ for $\mathrm{C}_{\mathrm{N}}=0.415$.

\section{Turbulence atmosphérique.}

Le calcul des différents organes d'une éolienne se heurte à une difficulté sérieuse. Si les condi- tions normales de fonctionnement et, en particulier, celles qui correspondent aux caractéristiques nominales sont bien définies, par contre, les conditions de marche accidentelles, au cours des- 
quelles apparaissent presque toujour's les efforts les plus grands, sont très mal connues. On définit, en général, une valeur du vent maximum que la machine est appelée à supporter. Outre ce qu'il y a d'arbitraire dans le choix de cette valeur maxima, il est bien évident que le problème est en fait beaucoup plus compliqué. Non seulement la valeur maxima de la vitesse du vent intervient, mais encore la rapidité des variations de vitesse, la fréquence et l'amplitude de ces variations, ainsi que la grandeur des variations de direction qui accompagnent les variations en intensité. Pour serrer encore de plus près le problème, il faudrait tenir compte des variations instantanées des caractéristiques du vent en chaque point de l'aire balayée par la roue de la machine, et établir la facon dont s'effectue dans la roue l'intégration des efforts variables en chaque point.

La résolution rigoureuse d'un tel problème est quasi impossible. On peut, tout au plus, espérer définir des conditions de marche représentant approximativement les limites à l'intérieur desquelles évolueront les efforts accidentels aléatoires. Une difficulté, et non des moindres, réside dans le fait que l'on ne dispose actuellement, à de rares exceptions près, que de mesures. anémométriques effectuées dans des conditions très différentes de l'emploi des éoliennes, et généralement dans un but assez différent (météorologie, étude de la turbulence fine de l'atmosphère). Les renseignements les plus fréquients sont donnés par les anémomètres enregistreurs. Indépendamment du fait que ceux-ci n'indiquent la valeur de la vitesse du vent qu'en un point (à l'échelle des éoliennes), les vitesses d'enregistrement sont telles qu'il est difficile d'utiliser leurs indications pour la connaissance des phénomènes rapidement variables qui intéressent les éoliennes. Dès lors, en dépit de l'existence d'une masse considérable d'enregistrcments anémométriques, une très petite quantité de ces enregistrements sont utilisables.

Etant dans l'impossibilité, par suite de la pauvreté actuelle des mesures, de tenir compte de l'hétérogénéité des vents dans l'aire balayée par les roues, on supposera que l'on a affaire à un vent moyen homogène, e'est-à-dire un vent ayant à un instant donné les mêmes caractéristiques en tout point de l'aire balayée. Nous nous proposons simplement de définir les grandeurs représentant les variations de ce vent, à savoir : intensité maximum, amplitude et fréquence des oscillations autour de la valeur moyenne, accélération, durée des rafales, écarts en direction.

La vitesse maximum de vent $\grave{a}$ considérer dépend évidemment de la région à laquelle elle s'applique. On la déduira du dépouillement des enregistrements anémométriques d'une période suffisamment longue s'étendant sur plusieurs années. Un tel maximum de vitesse apparaît toujours sous forme d'une pointe de très courte durée. C'est une oscillation de turbulence de grande amplitude qui se superpose à un vent moyen de valeur nettement plus faible. La durée très courte du vent maximum maximorum enregistré conduit à en tenir compte, d'une façon particulière, dans les calculs. Pour les régions européennes où l'exploitation de l'énergie éolienne est intéressante, et principalement pour la zone atlantique, on peut adopter une vitesse maximum de $55 \mathrm{~m} / \mathrm{s}$ mesurée à $10 \mathrm{~m}$ du sol.

Suivant la nature du sol environnant le point d'observation, ou plus exactement suivant son état de rugosité, les oscillations de turbulence qui se superposent au vent moyen seront plus ou moins développées. L'enregistrement dë cette turbulence donne une courbe très tourmentée avec une succession alternée de maxima et de

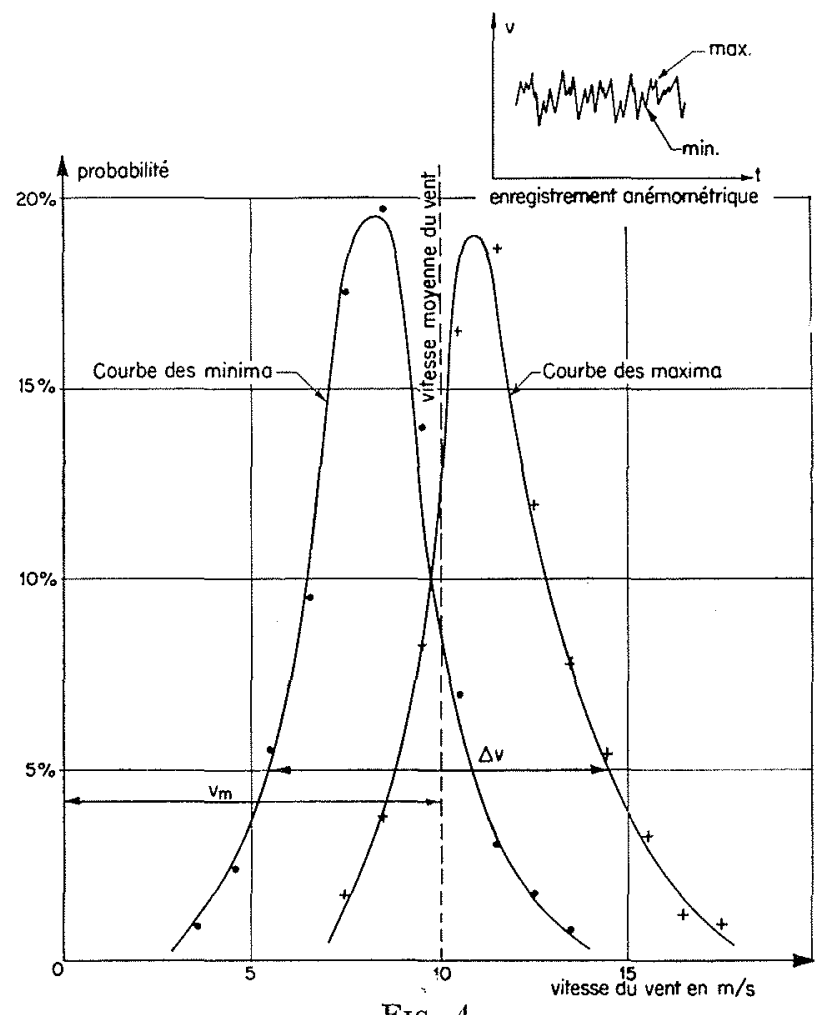

FIG. 4

minima. On caractérise assez bien un tel enregistrement de la manière suivante. En dénombrant d'une part les minima, d'autre part les maxima, et en déterminant le nombre de chacun d'eux qui appartient à une tranche donnée de vitesse de vent, on peut tracer deux courbes donnant respectivement la probabilité de rencontre des minima et des maxima atteignant une certaine valeur de la vitesse. On obtient ainsi deux courbes en cloche, situées de part et d'autre de la valeur moyenne de la vitesse. Ces courbes sont plus ou moins étalées suivant l'intensité de la tur- 
bulence. A chaque valeur de la probabilité $p$ correspond une valeur de l'écart de vitesse $\Delta v$ ou amplitude de la turbulence. Cette amplitude augmente lorsque la probabilité diminue. On peut adopter comme amplitude de la turbulence celle qui correspond, par exemple, à une probabilité de $5 \%$, et on caractérisera la turbulence du vent par le rapport $\Delta v / v_{m}$ de l'amplitude que l'on vient de définir, à la valeur moyenne de la vitesse. Ce rapport dépend du lieu choisi, il varie en général entre 0,6 et 1 , pour des mesures faites avec des anémomètres classiques des services météorologiques. Il est certain que le détail de la courbe d'enregistrement du vent dépend de la dimension de l'appareil de mesure et de son temps de réponse (c'est-à-dire de l'échelle de la turbulence observée), mais il est peu probable que les valeurs extrêmes qui interviennent pour définir $\Delta v$ varient beaucoup.

On peut se représenter la turbulence atmosphérique comme étant due ä la superpopsition de mouvements tourbillọnaires à un mouvement d'ensemble uniforme. Un tourbillon simple entraîné par le courant à vitesse $v_{m}$ donnera naissance à une oscillation de vitesse en même temps qu’à une oscillation de direction. Or, si les enregistrements anémométriques donnent facilement des indications sur les oscillations de vitesse, par contre, on ne dispose généralement d'aucune indication sur les oscillations de direction. On évaluera ces dernières par la règle suivante de composition des vitesses. On admet qu'une oscillation de vitesse $\Delta v$ est obtenue par la superposition à un courant $v_{m}$ d'un tourbillon de vitesse tangentielle uniforme égale à $\Delta v / 2$. Dans ces conditions, lorsque la vitesse tangentielle du tourbillon sera parallèle et de même sens que $v_{i m}$ on aura le maximum $v_{m}+\Delta v / 2$ et quand elle sera de sens contraire à $b_{m}$ on aura le minimum $v_{m}-(\Delta v / 2)$. Pour toutes les autres orientations de la vitesse tangentielle du tourbillon par rapport à la vitesse moyenne $v_{m}$, on obtiendra la vitesse résultante par la figure. Celle-ci permet, en particulier, de déterminer l'écart maximum en direction.

$$
\text { Soit } \mathrm{AB}=v_{m}-(\Delta v / 2) \text { et } \mathrm{AC}=v_{m}+(\Delta v / 2) \text {; }
$$

du point $A$, on mène les tangentes au cercle ayant $\mathrm{B} \mathrm{C}=\Delta v$ pour diamètre. Soit $\mathrm{A} \mathrm{D}$ et $\mathrm{A} \mathrm{E}$ ces tangentes; elles représentent les écarts maximum en direction. L'angle $\beta$ mesurant cet écart a pour valeur :

$$
\sin \beta=\frac{\Delta v}{2 \cdot v_{m}}
$$

C'est l'écart maximum en direction correspondant à une vitesse moyenne $v_{m}$ subissant une oscillation d'amplitude $\Delta v$.
Les observations donnent une assez bonne concordance avec les résultats déduits de la règle précédente.

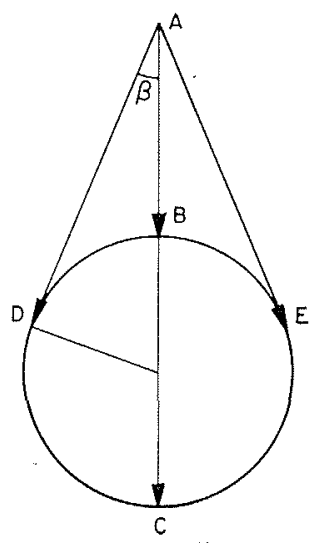

FIG. 5

Voici à titre d'exemple quelques chiffres rele-

\begin{tabular}{|c|c|c|c|}
\hline $\begin{array}{l}\text { VITESSE } \\
\text { MAXIMUM }\end{array}$ & $\begin{array}{l}\text { VITESSE } \\
\text { MINIMUM }\end{array}$ & $\begin{array}{l}\text { ECART } \\
\text { OBSER VÉ }\end{array}$ & $\begin{array}{c}\text { ECART } \\
\text { CALCULE }\end{array}$ \\
\hline $53 \mathrm{~m} / \mathrm{s}$ & $39 \mathrm{~m} / \mathrm{s}$ & $\pm 8^{\circ}$ & $\pm 9^{\prime \prime}$ \\
\hline 36,5 & 19,7 & $\pm 22^{\circ} 5$ & $\pm 24^{\prime \prime}$ \\
\hline 20,5 & 11 & $\pm 17^{\circ} 8$ & $=-19^{\circ}$ \\
\hline
\end{tabular}
vés à La Banne d'Ordanche:

Il est à noter que les écarts en direction se manifestent principalement dans un plan horizontal; ce sont des écarts en azimut; les écarts dans un plan vertical, c'est-à-dir'e en site, sont beaucoup plus faibles (de l'ordre de 7 à 10 fois plus faibles) et pratiquement négligeables, tant que l'on opère au voisinage d'un sol uni et horizontal. Il est évident qu'au voisinage d'un obstacle formant sillage, il n'en serait pas de même; mais cette disposition est toujours à éviter.

La connaissance des écarts en direction est de la plus grande utilité pour le calcul de la machine. En effet, compte tenu des moments gyroscopiques admissibles, il n'est pas possible de choisir une vitesse d'orientation suffisamment élevée pour suivre les écarts en direction; aussi les efforts les plus importants apparaissent-ils dans les pales lorsque, par suite d'une saute de vent, l'axe de la roue fait un certain angle avec la direction $\mathrm{du}$ vent. Ces efforts, variant et changeant de signe à chaque tour, soumettent la pale à des fatigues alternées dangereuses. Ce sont ces conditions qui imposent le choix de la structure de la pale, et non pas les fatigues en marche normale.

Il ne semble pas que, à l'échelle des phéno- 
mènes intéressant les aérogénérateurs, on rencontre d'accélération du vent supérieure à $4 \mathrm{~m} / \mathrm{s}^{2}$. En prenant ce chiffre comme base, on obtient la durée minimum des accroissements de vitesse; celle-ci augmente quand la vitesse moyenne du vent augmente. Or, les écarts maximum en direction s'obtenant dans le temps mis pour franchir l'écart de vitesse $\Delta v$, on en déduit que les variations de direction sont plus lentes par vent fort que par vent faible, ce qui est bien vérifié par l'expérience.

La loi de répartition de la vitesse du vent suivant la verticale joue un grand rôle tant pour le calcul des efforts que pour le choix des dimensions optimum à donner aux machines. On la rencontre dans les problèmes suivants :

- Répartition de l'énergie utilisable dans la surface balayée par la roue;

- Modification cyclique des efforts sur les pales;

- Déplacement du centre de poussée;

- Influence sur le rendement aérodynamique des variations cycliques d'incidence du courant relatif;

- Influence sur la variation du poids et du prix des machines par $\mathrm{kWh}$ produit, en fonction des dimensions de la roue.

La loi de répartition des vitesses suivant une verticale dépend, d'une part du relief local au point considéré, d'autre part de la rugosité des régions sur lesquelles le vent a soufflé. Il est connu qu'au sommet d'une colline arrondie on obtient un accroissement local de vitesse, dont l'effet peut être tel qu'il inverse le sens du gradient de vitesse habituel, en sorte que, contrairement au résultat classique, on peut obtenir au voisinage du sol une vitesse plus grande qu'à une certaine hauteur. Cette particularité mise à part, en général, en terrain plat. on peut représenter la variation de vitesse $v$, en fonction de la hauteur $h$ au-dessus du sol, par une loi de la forme:

$$
v=v_{o}\left(h / h_{o}\right)^{1 / n}
$$

$v_{0}$ étant la vitesse à la hauteur $h_{o}$ et $n$ ayant une valeur variant dans de notables proportions, suivant la rugosité des régions balayées par le vent. Un exemple typique de cette influence est donné par le fait suivant. Lorsque la mesure de répartition en altitude est faite au bord de la mer, on trouve un résultat très différent suivant que le vent vient de la mer ou de la terre. Pour le vent de mer, la variation avec l'altitude est très faible, c'est-à-dire que $n$ est grand, cependant que, pour le vent de terre, le gradient est important et $n$ diminue. Pour un terrain horizontal avec rugosités normales, $n$ ne descend pas audessous de 5. Au cours de l'étude exposée plus loin, on a adopté la valeur $n=5$, valeur d'ailleurs favorable à l'adoption de machines de grandes dimensions.

Un élément très important pour les calculs de résistance des aérogénérateur's est la connaissance de la courbe donnant pour les grandes vitesses (voisines du maximum) la durée correspondant à chaque vitesse. La courbe habituelle vitesse-durée établie pour les calculs d'énergie est difficilement utilisable dans ce cas, car, d'une part, elle n'est généralement pas assez précise aux grands vents, son échelle de temps est trop resserrée, d'autre part, elle est établie pour des vents moyens et non pas pour les détails de la turbulence. Supposons une telle courbe établie en tenant compte des oscillations de turbulence, elle aura l'allure de la figure 6. La pointe, ou

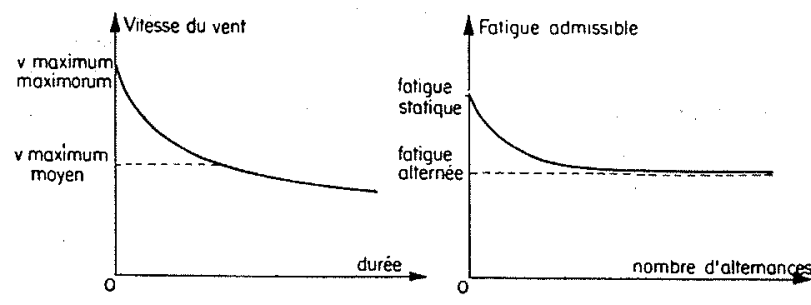

FII. 6

vitesse maximum maximorum, est l'écart maximum obtenu à partir d'un vent maximum moyen. Les durées, d'abord très faibles, croissent rapidement lorsqu'on atteint la zone des vitesses moyennes. L'échelle des abscisses peut être graduée en nombre de tours de la roue, en sorte qu'il est possible, grâce à cette courbe, de connaître pour chaque vitesse de vent le nombre d'alternances d'efforts subis par la roue. Il convient de mettre en parallèle avec cette courbe la courbe donnant, pour le matériau constituant la rove, la variation de la fatigue admissible en fonction du nombre d'alternance des efforts. Les deux courbes ont, en gros, la même allure. Cette particularité remarquablè permet d'utiliser, pour les calculs des efforts au vent maximum maximorum, des fatigues admissibles nettement plus élevées que pour les vents moyens. La comparaison des courbes de vitesse de vent et de fatigues admissibles, en fonction du nombre d'alternances, permet de déterminer quelle vitesse de vent conduit aux sections maximum; ce n'est pas forcément le vent maximum maximorum. En tout cas, la similitude d'allure des courbes indiquées montre qu'il n'y aura pas d'écart considérable entre les sections obtenues en calculant la machine pour le vent maximum maximorum ou pour un vent moyen. Cette circonstance est évidemment très favorable. 


\section{Choix du vent nominal.}

L'énergie éolienne disponible en un lieu donné sera représentée de la façon la plus parlante au moyen de la courbe vitesse-durée donnant pour chaque valeur de la vitesse du vent le nombre d'heures par an, pendant lesquelles on dispose d'un vent dont la vitesse est au moins égale à la valeur considérée. Pour la plupart des régions où des relevés anémométriques ont permis d'établir de telles courbes, on constate que l'allure des courbes vitesse-durée est la même. $\mathrm{Si}$, pour toutes les courbes considérées, on choisit une échelle des ordonnées telles que soient confondus

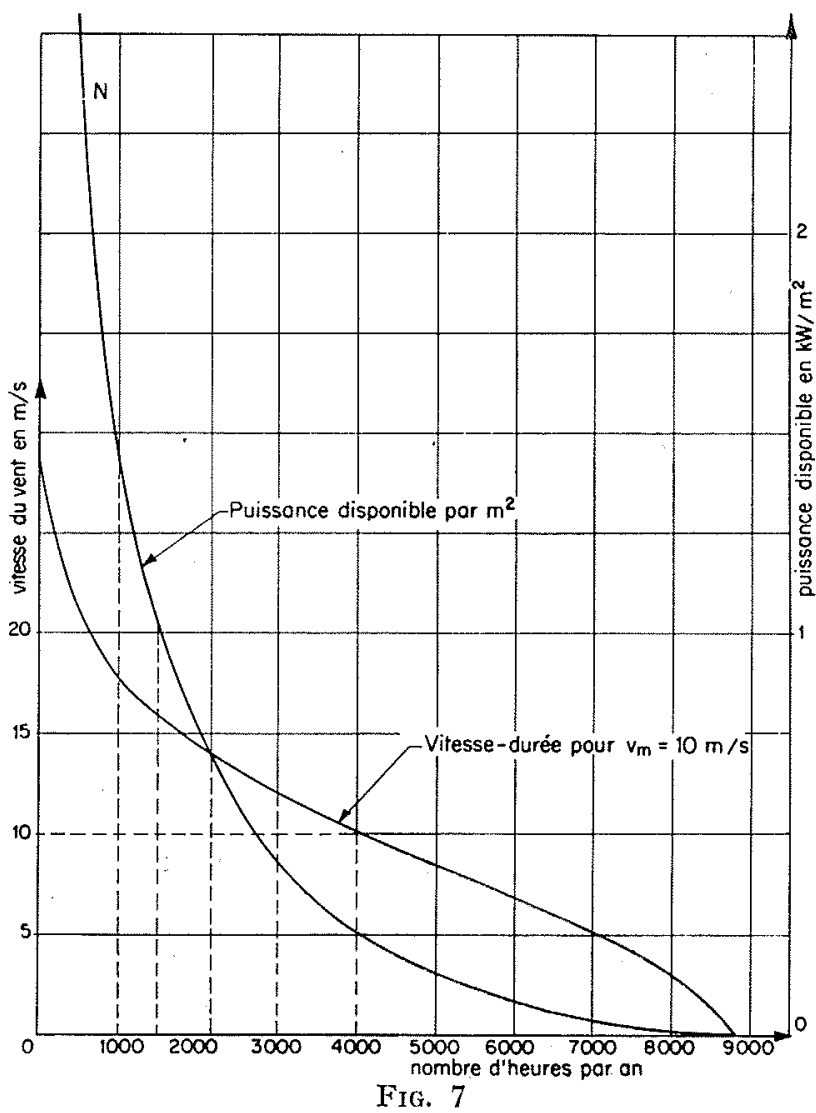

les points correspondant, par exemple, à la durée de 4.000 heures par an, on constate que les différentes courbes viennent se situer approximativement sur un même tracé. A un changement de l'échelle des ordonnées près, on peut donc, en première approximation, admettre qu'une seule courbe peut représenter des sites différents. Le changernent d'échelle sera simplement défini par la valeur du vent moyen dans l'année, qui est d'ailleurs la valeur du vent pour une durée de 4.000 heures. La courbe des vitesses-durées permet d'établir une courbe donnant, en fonction du nombre d'heures, la puissance disponible. L'aire située sous cette courbe indiquera l'énergie disponible dans l'année. Mais l'aérogénérateur ne peut pas donner toute l'énergie ainsi théoriquement disponible dans le vent; différentes considérations interviennent pour limiter cette énergie.

Lorsque la vitesse du vent croìt, la machine ne commence à débiter que pour une certaine vitesse dite 《vitesse d'amorçage », vitesse telle que la puissance disponible sur l'arbre de la machine corresponde à la puissance absorbée par les pertes à vide de l'ensemble du groupe (pertes génératrice, pertes multiplicateur, pertes par les auxiliaires). Puis, la vitesse du vent croissant, on atteint une certaine valeur dite « vitesse de vent nominale $»$ qui est la vitesse par laquelle la puissance nominale de la machine est atteinte. Pour toute valeur de la vitesse supérieure à la vitesse nominale, la puissance sera maintenue constante par le système de réglage. Enfin, la vitesse $\mathrm{du}$ vent continuant à crôtre, elle atteint une valeur dite "de mise hors service », audelà de laquelle la machine est arrêtée et ne débite plus, pour des raisons de sécurité.

L'aire correspondant à l'énergie produite dans l'année sera donc limitée :

$1^{\circ}$ à la partie inférieure, par une horizontale d'or-

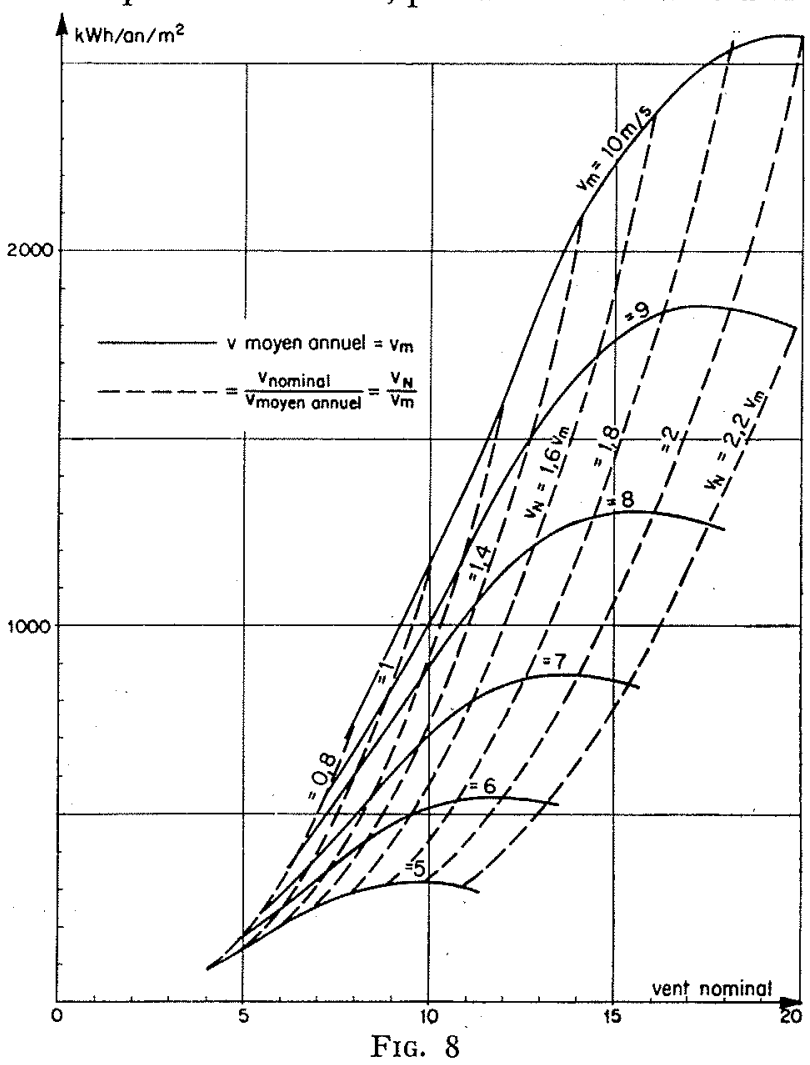

Energie produite en $\mathrm{kWh} / \mathrm{m}^{2} / \mathrm{an}$ en fonction de la vitesse du vent nominale, pour différents vents moyens annuels.

Energy produced in $\mathrm{kWh} / \mathrm{m}^{2} /$ year as a function of the rated wind speed, for different annual average wind speeds. 
donnée égale à la puissance correspondant au vent «d'amorçage »,

$2^{\circ}$ à droite, par la courbe puissance-durée,

$3^{\circ}$ à la partie supérieure, par une horizontale d'ordonnée égale à la puissance nominale,

$4^{\circ}$ à gauche, par une verticale dont l'abscisse correspond à la durée des vents de mise hors service.

Cette dernière quantité est pratiquement indépendante du choix des caractéristiques de la machine.

La grandeur essentielle à déterminer est la valeur du vent nominal, ou de la puissance nominale. Quant à la vitesse d'amorcage et aux pertes à vide, elles dépendent de la valeur de la puissance nominale.

On a admis dans les calculs ayant servi à la détermination des courbes de la figure 8 que la puissance totale absorbée par les pertes à vide du groupe était égale. à $10 \%$ de la puissance nominale. On a admis, d'autre part, que la durée de mise hors service par grand vent était uniformément de 250 heures dans l'année.

Dans ces conditions, on constate que pour une vitesse moyenne du vent dans l'année (c'est-à-dire pour une courbe vitesse-durée caractérisant un site), la courbe représentant les variations de l'énergie produite dans l'année en fonction de la valeur du vent nominal (c'est-à-dire en fonction de la puissance nominale de la machine), est d'abord croissante, puis présente un maximum et finalement retombe lorsque le vent nominal continue à augmenter. Il y a donc, pour chaque valeur du vent moyen, une valeur du vent nominal qui donne le maximum d'énergie possible. Ce maximum est obtenu lorsque l'on a:

vent nominal $=1,9 \times$ vent moyen annuel.

L'existence du maximum est due au fait que les pertes à vide du groupe croissent lorsque la puissance nominale augmente, cependant que le nombre d'heures de fonctionnement diminue.

Du point de vue économique, on devra choisir pour l'aérogénérateur une puissance nominale correspondant à un vent nominal inférieur à celui qui vient d'être indiqué, car ce qui importe, c'est avant tout d'obtenir le prix de revient minimum pour le $\mathrm{kWh}$ produit. Or, dans le prix total de l'installation éolienne, une part des charges augmente avec la puissance équipée. Par suite de cette croissance, l'optimum économique s'obtiendra pour une vitesse nominale inférieure à 1,9 fois la vitesse du vent moyen annuel. Finalement, la valeur du vent nominal dépendra de l'importance relative, par rapport au prix total de l'installation, des charges croissant avec la puissance équipée. Nous adopterons, comme une bonne approximation du prix total, une formule de la forme:

$$
\mathrm{P}_{i}=\mathrm{P}\left[(1-\alpha)+\alpha\left(\frac{v_{n}}{1,5 v_{\text {moy }}}\right)^{3}\right]
$$

$\mathrm{P}_{i}$ étant le prix total pour un équipement correspondant à une valeur $v_{n}$ du vent nominal.

$P$ le prix total pour un équipement tel que $v_{n}=1,5 \mathrm{v}_{\text {moy, }}$.

a représente la part des charges proportionnelles à la puissance équipée (alternateurs, transformateurs, lignes, mécanismes de réglage, etc.).

Dans ces conditions, on constate que pour une valeur donnée de $\approx$ (c'est-à-dire pour un type de construction), le prix de revient du $\mathrm{kWh} / \mathrm{an}$ passe par un minimum pour une valeur donnée de la vitesse du vent nominale, c'est-à-dire pour

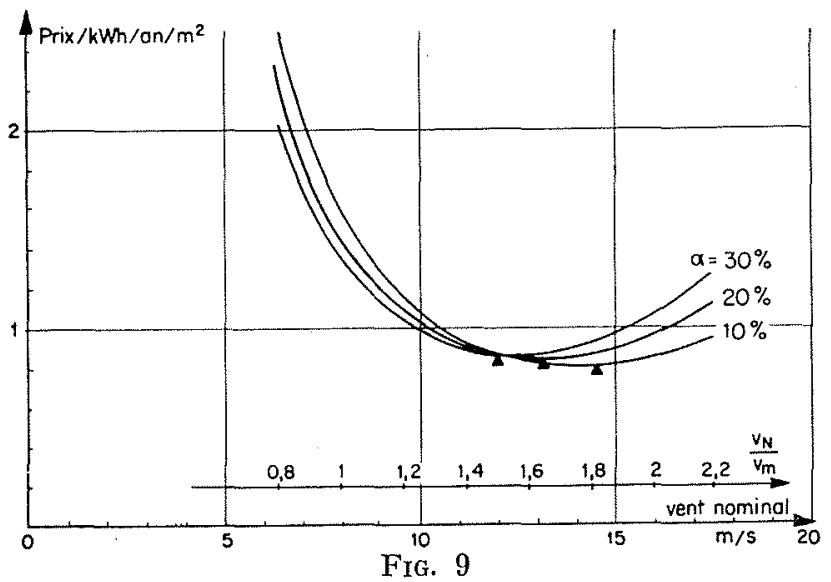

Variat ion avec le vent nominal du prix du $\mathrm{kWh} / \mathrm{m}^{2} /$ an pour différentes valeurs de $\alpha$, le prix d'une machine de $1.000 \mathrm{~m}^{2}$ avec $\alpha=30 \%, v_{n} / v_{m}=1,5$ et $v_{m}=8 \mathrm{~m} / \mathrm{s}$, étant pris pour unité. Toutes les courbes sont établies pour $v_{m}=8 \mathrm{~m} / \mathrm{s}$ avec

$$
\operatorname{Prix}=\mathrm{P}\left[(1-\alpha)+\alpha\left(v_{n} / 1,5 v_{m}\right)^{3}\right]
$$

Variation, with the rated wind speed, of the cost per $\mathrm{kWh} / \mathrm{m}^{2} /$ year for different values of a, the cost of a machine of $1,090 \mathrm{~m}^{2}$ with $\alpha=30 \%, \mathrm{v}_{\mathrm{n}} / \mathrm{v}_{\mathrm{m}}=1.5$ and $\mathrm{v}_{\mathrm{m}}=8 \mathrm{~m} / \mathrm{s}$ being taken as unity. All the curves are drawn for $\mathrm{v}_{\mathrm{m}}=8 \mathrm{~m} / \mathrm{s}$ with

$$
\text { Cost }=\mathbf{P}\left[(1-\alpha)+\alpha\left(v_{n} / 1,5 v_{m}\right)^{3}\right]
$$

une valeur donnée de la puissance nominale. On définit ainsi l'équipement en puissance constituant l'optimum économique pour un type donné de construction. Si on définit la valeur du vent nominal par rapport à la vitesse moyenne annuelle, le résultat sera indépendant du site. On obtient ainsi :

$$
\begin{array}{lll}
\alpha=0,3 & v_{n}=1,5 & v_{\mathrm{moy}} \\
\alpha=0,2 & v_{n}=1,65 & v_{\mathrm{moy}} \\
\alpha=0,1 & v_{n}=1,8 & v_{\mathrm{moy}} \\
\alpha=0 & v_{n}=1,9 & v_{\mathrm{moy}}
\end{array}
$$


A titre d'exemple, une machine de $25 \mathrm{~m}$ de diamètre, caractérisée par $\alpha=0,2$ et devant fonctionner dans une région où la vitesse du vent moyen annuel est de $8 \mathrm{~m} / \mathrm{s}$, devra être équipée pour un vent nominal $v_{n}=1,65 \times 8=13,2 \mathrm{~m} / \mathrm{s}$, c'est-à-dire pour une puissance de $290 \mathrm{~kW}$ sur l'arbre. Dans ces conditions, cette machine produira une énergie annuelle de $610.000 \mathrm{kWh}$.

\section{Différentes dispositions possibles pour la construction de la machine.}

Dans ses grandes lignes, l'éolienne pour production d'énergie électrique sera une machine rapide à faible nombre de pales et entraînement de la génératrice par multiplicateur à engrenages. Les progrès accomplis dans la construction des multiplicateurs, d'une part, le prix élevé et les difficultés de construction des génératrices à très faible vitesse, d'autre parrt, conduisent à éliminer l'attaque directe de la génératrice par la roue, même dans le cas de grandes puissances. Dans le cadre ainsi défini, plusieurs solutions se présentent sur les points suivants :

NOMBRE de PALES de la ROUE : le choix portera sur les dispositions à deux ou trois pales. La solution à plus de trois pales ne se justifie pas, étant donné l'intérêt des vitesses de rotation élevées; quant à la solution à une pale qui a été utilisée pour de très petites machines, elle ne paraît pas devoir être retenue, par suite des difficultés d'équilibrage.

Montage des pales sur le noyau : les deux solutions à pales orientables et à pales fixes ont élé également étudiées. Des réalisations des deux types ont fonctionné.

POSITION DE LA ROUE : celle-ci peut être placée soit à l'amont, soit à l'aval du pylòne. Si la première position paraît préférable pour éviter l'effet de sillage du pylòne, la deuxième, par contre, bénéficie d'un mécanisme d'orientation moins coûteux, par suite de la diminution des efforts de manœuvre.

GÉNératrices Électrioues : deux solutions sont en présence : la génératrice asynchrone et la génératrice synchrone avec excitatrice. La rusticité de la première machine et la facilité de son accrochage sur le réseau sont des atouts précieux. Par contre, l'obligation de prélever le courant magnétisant sur le réseau pose des problèmes dans le cas d'une grande puissance équipée en énergie éolienne avec machine asynchrone, et redonne de l'intérêt à la solution synchrone.

Indépendamment du choix de ces différentes solutions, restent à préciser les caractéristiques principales : puissance, hauteur du pylône, vitesse de rotation.

Nous allons examiner plus en détail les principaux problèmes que nous venons de poser.

\section{Problèmes posés par la construction des pales.}

Du point de vue étude mécanique et construction, le problème le plus délicat de l'aérogénérateur est posé par la réalisation des pales. Si les autres organes font appel à des techniques éprouvées et à des constructions classiques, les pales, par contre, tant par leurs dimensions que par les conditions très particulières de fatigue auxquelles elles sont soumises, nécessitent des études particulières et des solutions nouvelles. Contrairement à ce que l'on croit fréquemment, ce n'est d'ailleurs pas dans le domaine de l'aérodynamique que réside la difficulté, mais bien dans celui de la construction et de la résistance des matériaux. Des méthodes classiques permettent de tracer des hélices dont les rendements sont suffisamment bons pour qu'il n'y ait pas grandchose à gagner à chercher à les perfectionner; nous n'y reviendrons donc pas. Le mode de réalisation des pales, par contre, peut faire l'objet d'améliorations qui se traduiront par une plus grande sécurité de marche et une plus grande économie de fabrication.

Il est évident qu'il y a intérèt à utiliser des hélices à grande vitesse spécifique pour réduire tant le poids de la roue elle-même que celui du multiplicateur. Le choix de la vitesse spécifique de la roue peut ètre considéré comme primordial, puisqu'en définitive il commande le nombre de pales, le moment d'inertie de la roue, les fréquences propres de vibration et jusqu'aux caractéristiques de fonctionnement. C'est donc de ce probleme particulier que nous nous occuperons.

Nous adopterons pour le calcul de la pale la méthode de Stefaniak (Windrad grösster Leistungsabgade - Forschung, Bd. 19, H. 1). On appelle :

$\mathrm{C}_{a}=$ coefficient de portance des profils;

$\mathrm{C}_{w}=$ coefficient de traînée;

$l=$ longueur de corde;

$u=$ vitesse tangentielle;

$v=$ vitesse du vent;

$w=$ vitesse relative;

$\mathrm{R}=$ rayon;

$z=$ nombre de pales;

$\mathrm{L}=$ longueur d'un élément de pales;

$A=$ portance; 


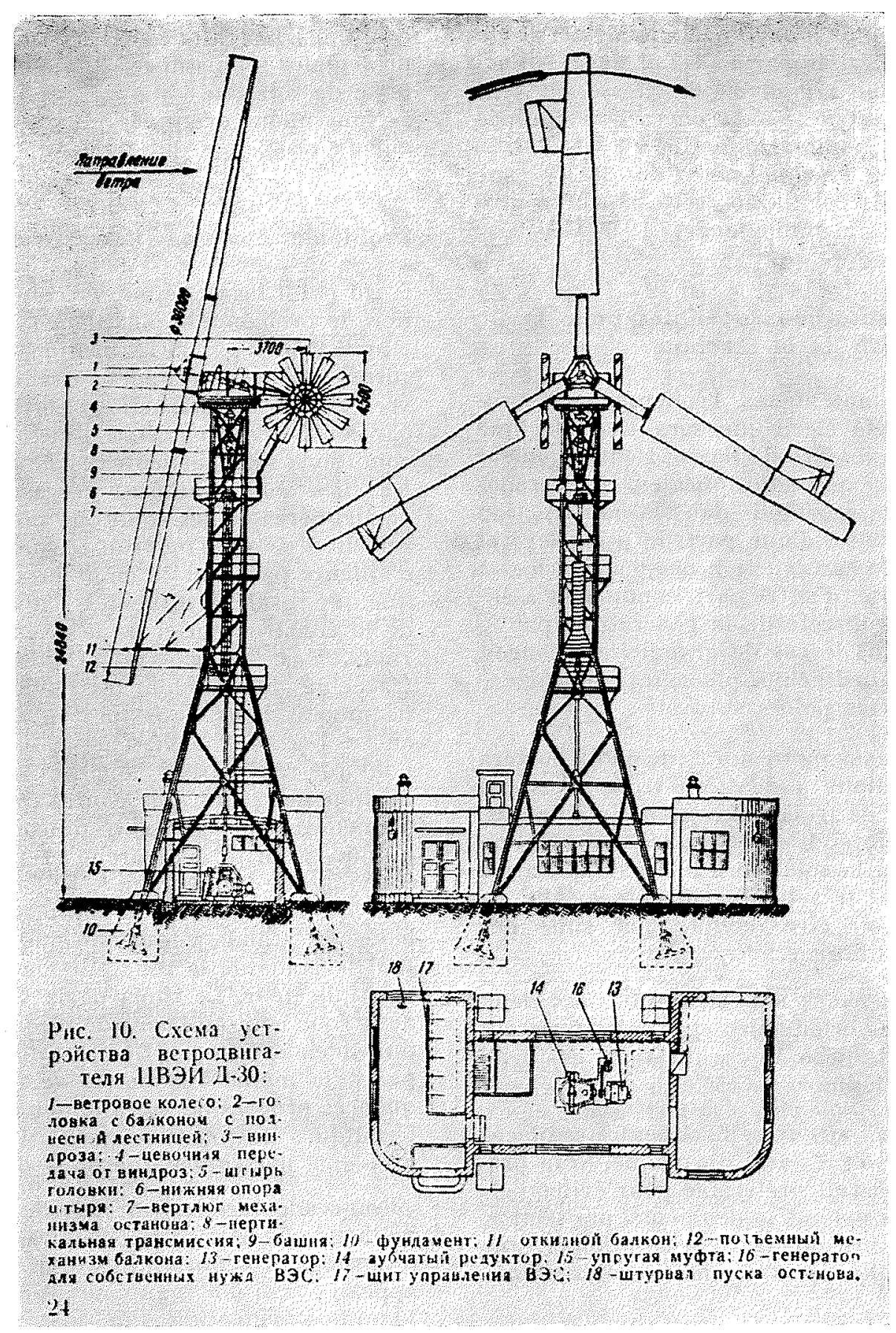

Aérogénérateur ZWEI-D-30. Roue de $30 \mathrm{~m}$ de diamètre à pales partiellement orientables, système Sabinin-Krasnowski. Par vent de $8 \mathrm{~m} / \mathrm{s}$, cette machine donne $50 \mathrm{~kW}$ a $25 \mathrm{tr} / \mathrm{mn}$. Le caractère dominant de la construction est la rusticité. (Extrait de l'ouvrage de Sidorov Vetrodvigateli v Artike.)

$Z$ wei-D-30 aerogenerator. Rotor of $30 \mathrm{~m}$ diameter with blades partially variable in pitch, Sabinin-Krasnowski design. In a wind of $8 \mathrm{~m} / \mathrm{s}$, this machine gives $50 \mathrm{~kW}$ at $25 \mathrm{r} . \mathrm{p} . \mathrm{m}$. The principal feature of the construction is its robust quality. (Extract from the work of Sidorov Vetrodvigateli

$\gamma=$ poids spécifique de l'air;

$I=$ moment d'inertie de la section;

$\sigma=$ fatigue.
Pour une section de la pale située à la distance $R$ de l'axe, on a la relation:

$$
\mathrm{C}_{a} . l . z=\frac{\mathrm{R}}{u / v} \cdot \Lambda
$$


$\Lambda$ étant une grandeur sans dimension donnée par le tableau suivant, en fonction du rapport $u / v:$

\begin{tabular}{|c|c|c|c|c|c|c|c|c|}
\hline$u / v=$ & 1 & 2 & 3 & 4 & 5 & 6 & 7 & 8 \\
\hline & 3,36 & 2.38 & 1,73 & 1,33 & 1,08 & 0,91 & 0,8 & 0,7 \\
\hline$u / v=$ & 9 & 10 & 11 & 12 & 13 & 14 & 15 \\
\hline & - & $-0,62$ & 0,56 & 0,50 & 0,46 & 0,42 & 0,39 & 0,37 \\
\hline
\end{tabular}

Pour une roue de diamètre donné et de vitesse de rotation donnée, on a ainsi la valeur du produit $\mathrm{C}_{a} . l . z$ le long de la pale.

Le graphique figure 11 indique, en fonction du

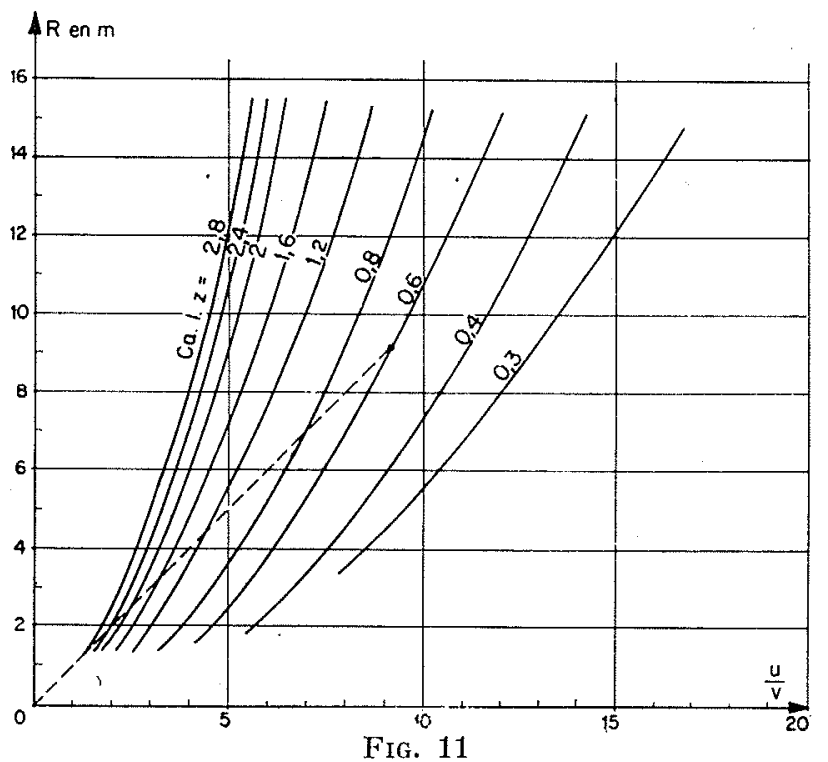

rapport $u / v$ de la vitesse tangentielle à la vitesse du vent et de la distance à l'axe, la valeur du produit $\mathrm{C}_{a} . l . z$. Ce graphique, d'emploi général, permet immédiatement de déterminer l'évolution des profils le long d'une pale quelconque; en effet, tous les points correspondant à une même pale sont situés sur un même segment de droite passant par l'origine, et dont l'extrémité est définie par le rayon de la pale et par son rapport $u / v$ périphérique. Sur la figure 11 est indiqué, à titre d'exemple, le segment correspondant à une pale de $9 \mathrm{~m}$ de rayon et ayant un $u / v$ périphérique de 9 .

Supposons que l'on adopte toujours le même profil dans les mêmes conditions d'incidence et de finesse : soit le profil Göttingen 624 (profil donnant la meilleure finesse pour le maximum d'épaisseur relative) utilisé à sa plus grande finesse $\mathrm{C}_{v} / \mathrm{C}_{a}=2 / 100$, incidence $3^{\circ}$ et $\mathrm{C}_{a}=0,92$; on obtient alors immédiatement la longueur de corde à la section considérée, le nombre de pales étant supposé choisi. La portance A d'un élément de pale de longueur $\mathrm{L}$ s'écrit alors :

$$
\mathrm{A}=\mathrm{C}_{a} \frac{\gamma w^{2}}{2 g} \cdot l . \mathrm{L}
$$

On peut ainsi déterminer le long de la pale envisagée la répartition de la portance et, par suite, la répartition du moment fléchissant $\mathrm{M}_{f}$. Les sections étant connues tout au long de la pale, on en déduit les moments d'inertie et, finalement, les fatigues.

Pour le profil Göttingen 624 considéré, de $160 \mathrm{~mm}$ d'épaisseur maximum pour une corde

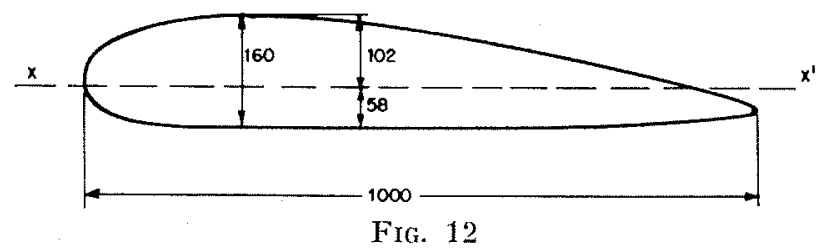

de $1.000 \mathrm{~mm}$, l'axe neutre longitudinal $\mathrm{XX}^{\prime}$ est à $58 \mathrm{~mm}$ de l'intrados. Le moment d'inertie autour de cet axe a la valeur suivante :

$I_{r}=20.000 \mathrm{~cm}^{4} \quad$ section $1.100 \mathrm{~cm}^{2}$, pour un profil plein.

Pour un profil creux d'épaisseur $e$, si $e=4 \mathrm{~cm}, \mathrm{I}_{x x}=18.400 \mathrm{~cm}^{4}$ et la section $=880 \mathrm{~cm}^{2}$ si $e=2 \mathrm{~cm}, \mathrm{I}_{x \cdot x^{*}}=13.750 \mathrm{~cm}^{4}$ et la section $=440 \mathrm{~cm}^{2}$ si $e=1 \mathrm{~cm}, \mathrm{I}_{x: x}=8.500 \mathrm{~cm}^{4}$ et la section $=220 \mathrm{~cm}^{2}$

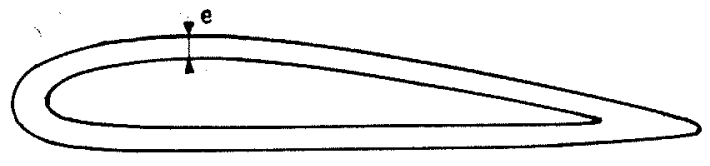

Fra. 13

a) CAS OÙ L'AXE DES PALES EST PERPENDICULAIRE A L'AXE DE ROTATION DE LA ROUE :

Partant de ces éléments, il est possible de déterminer la fatigue dans la pale en marche normale. On a effectué des calculs dans les hypothèses suivantes:

- Fonctionnement normal par vent constant, sans gradient, de $12 \mathrm{~m} / \mathrm{s}$;

- Axe des pales perpendiculaires à l'axe de rotation;

— Force centrifuge négligée;

- Profil à section pleine;

- Roue bipale et tripale. 
On constate aisément que, pour des roues homothétiques ayant même valeur de $u / v$ et fonctionnant dans un même vent, les fatigues en des points homologues sont identiques. Les résultats obtenus sont donc indépendants des dimensions de la roue. On a représenté alors sur un graphique (fig. 14) les fatigues obtenues en fonction de la valeur du rapport $u / v$ périphérique et de la distance à l'axe, le rayon de la roue étant pris comme unité.
On constate que la fatigue croit rapidement lorsque le rapport $u / v$ augmente, ce qui, avec la baisse de rendement, vient limiter les possibilités de réalisation des roues rapides. En outre, le maximum de fatigue dans la pale n'est pas obtenu au pied, mais à une distance de l'axe égale sensiblement à 0,6 fois la longueur de la pale. Pour des roues tripales, les fatigues maximums obtenues sont les suivantes :

\begin{tabular}{|c|c|c|c|c|c|c|c|c|c|c|c|c|}
\hline & $u / v=$ & 5 & 6 & 7 & 8 & 9 & 10 & 11 & 12 & 13 & 14 & 15 \\
\hline Section pleine : & $\begin{array}{l}\sigma_{\max }= \\
\left(\mathrm{kg} / \mathrm{cm}^{2}\right)\end{array}$ & 26 & 80 & 200 & 450 & 900 & 1.635 & 2.800 & 4.500 & 7.500 & 12.000 & 16.350 \\
\hline $\begin{array}{l}\text { Section creuse, épais } \\
\text { seur égale à } 1 / 10 \text { de } \\
\text { la hauteur du profil : }\end{array}$ & $\sigma_{\max }=$ & 43 & 132 & 330 & 740 & 1.420 & 2.700 & 4.650 & 7.400 & 12.400 & 19.900 & 27.000 \\
\hline
\end{tabular}

Pour les roues bipales de mème valeur de $u / v$ périphérique, les fatigues sont égales à 0,45 fois les fatigues des roues tripales, d'où l'intérêt des roues bipales aux grandes vitesses.

Les fatigues que nous venons de déterminer sont obtenues en marche normale; toutefois, des efforts plus importants seront supportés, en particulier, lors des écarts en direction aux grands vents; la majoration correspondante est d'ailleurs moins sensible aux grandes valeurs de $u / v$. On peut considérer que, pour un écart en direction de $40^{\circ}$ par vent de $40 \mathrm{~m} / \mathrm{s}$ et dans le cas de pales orientables, la majoration est de 1,5 pour $u / v=5,1,25$ pour $u / v=10$ et 1,15 pour $u / v=15$. Il faut, en outre, tenir compte des efforts gyroscopiques d'orientation. En admettant une vitesse d'orientation identique pour toutes les machines, la fatigue gyroscopique peut être prise égale à 0,4 fois la fatigue normale pour $u / v=5$, a 0,06 fois la fatigue normale pour $u / v=10$ et à 0,017 pour $u / v=15$. Finalement, les fatigues maximums supportées par la pale se déduiront des fatigues de marche normale précédemment calculées en multipliant par un coefficient $\mathrm{X}$ dépendant de $u / v$. Pour une roue tripale, on obtient les valeurs suivantes :

\begin{tabular}{|c|c|c|c|c|c|c|c|c|c|c|c|c|}
\hline & $u / v=$ & 5 & 6 & 7 & 8 & 9 & 10 & 11 & 12 & 13 & 14 & 15 \\
\hline & $\mathrm{x}=$ & 1,9 & 1,71 & 1,56 & 1,46 & 1,38 & 1,31 & 1,26 & 1,23 & 1,20 & 1,18 & 1,167 \\
\hline Section pleine : & $\begin{array}{c}\sigma_{\max }= \\
\left(\mathrm{kg} / \mathrm{cm}^{2}\right)\end{array}$ & 49,5 & 137 & 312 & 655 & 1.240 & 2.130 & 3.520 & 5.500 & 9.000 & 14.200 & 19.000 \\
\hline Section creuse : & $\sigma_{\max }=$ & 82 & 226 & 520 & 1.090 & 2.050 & 3.550 & 5.820 & 9.150 & 14.900 & 23.500 & 31.500 \\
\hline
\end{tabular}

Pour une bipale, ces valeurs sont à multiplier par 0,45 .

Reste à examiner le cas où la roue est arrêtée pales faces au vent par les vents les plus violents. La pale est alors assimilée à une plaque plane dont la forme est celle de la projection de la pale sur un plan perpendiculaire à l'axe de la roue. Le calcul est fait en adoptant comme vent maximum une vitesse de $63 \mathrm{~m} / \mathrm{s}$ (valeur correspondant, pour une hauteur de $20 \mathrm{~m}$, à un vent de $55 \mathrm{~m} / \mathrm{s}$ à $10 \mathrm{~m}$ du sol pour un gradient en $\left.\mathrm{H}^{1 / 5}\right)$. Comme précédemment pour des roues homothétiques, les fatigues en des points homologues sont identiques. On représentera donc les 


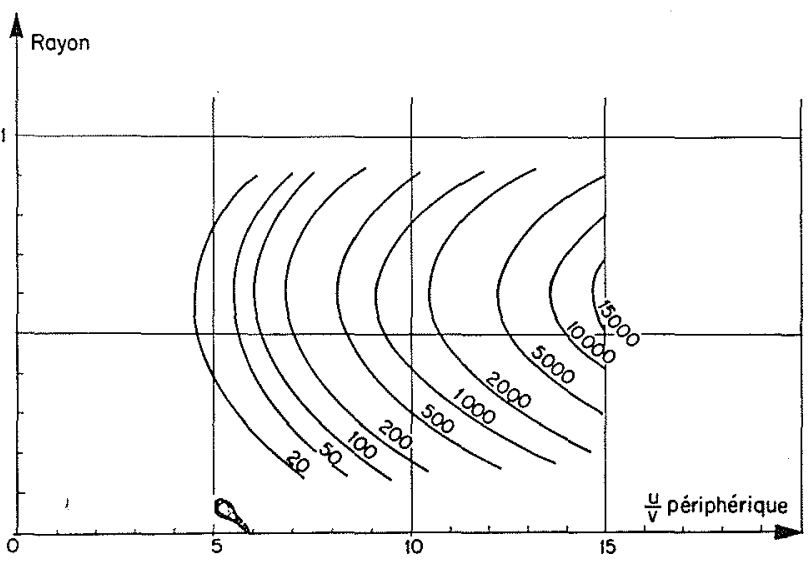

FIG. 14

Fatigue, en $\mathrm{kg} / \mathrm{cm}^{2}$, dans une pale en marche normale par vent de $12 \mathrm{~m} / \mathrm{s}$. Rone tripale, profil plein. (Il n'est tenu compte dans ce graphique ni des efforts d'orientation, ni des effets de turbulence.)

Stress, in $\mathrm{kg} / \mathrm{cm}^{2}$, in a blade dî̀ring normal operation with a vind of $12 \mathrm{~m} / \mathrm{s}$. Three-bladed rotor, solid section. (No account is taken in this graph either of the yawing stresses or of the effects of turbulence.)

résultats du calcul sur un graphique (fig. 15) donnant les fatigues en fonction du rapport $u / v$ périphérique et de la distance à l'axe, le rayon de la roue étant pris comme unité. Le graphique est établi pour une roue tripale. Pour une roue bipale, les valeurs indiquées sont à multiplier par 0.45 .

On constate ici encore que, par suite de la forme de la pale, les fatigues maximums ne sont pas atteintes au pied de la pale, mais à une distance de l'axe approximativement égale à 0,6 fois la longueur totale. La comparaison des graphique des figure 14 et figure 15 montre que, aux valeurs faibles de $u / v$, la fatigue à l'arrêt face au vent maximum est plus importante que la fati-

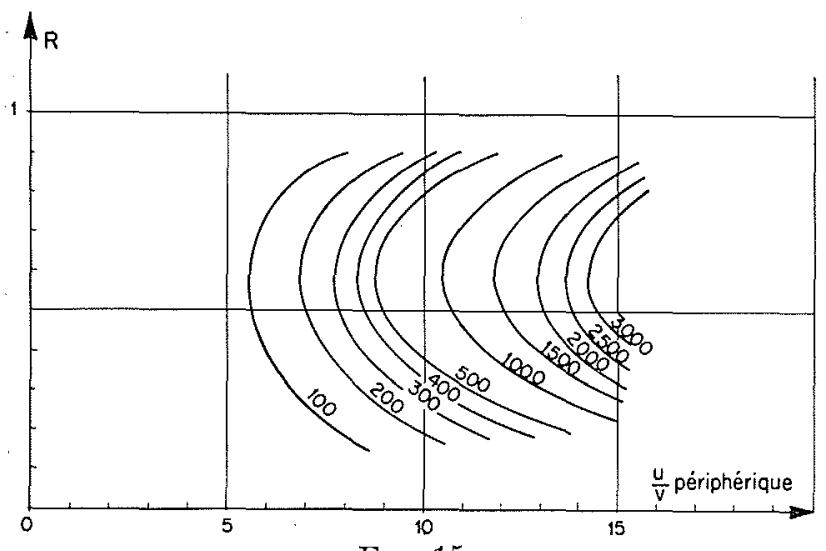

FIG. 15

Fatigue, en $\mathrm{kg} / \mathrm{cm}^{2}$, dans une pale arrêtée face an vent, par vent de $63 \mathrm{~m} / \mathrm{s}$. Roue tripale, profil plein.

Stress, in $\mathrm{kg} / \mathrm{cm}^{2}$, in a blade at standstill facing a wind of $63 \mathrm{~m} / \mathrm{s}$. Three-bladed rotor, solid section. gue en marche normale, mais très rapidement, lorsque $u / v$ augmente, la fatigue en marche normale l'emporte, en sorte que la fatigue à l'arrêt face au vent maximum n'intervient pas pour limiter les valeurs de $u / v$ admissibles. Si on tient compte, d'une part, de l'augmentation des fatigues dans la pale, d'autre part, de la diminution du rendement $\left(^{\star}\right)$, il ne paraît pas possible, dans l'état actuel de la technique, de réaliser économiquement des pales dont le rapport $u / v$ dépasse 9. Il semble même que la valeur la plus intéressante soit nettement inférieure, et comprise entre 5 et 7 suivant le type de construction adopté.

b) Cas ou l'axe des pales fait aVec L'AXe De LA ROUE UN ANGLE DIFFÉRENT DE $90^{\circ}$.

Cette disposition, appelée généralement disposition en « dièdre », permet, par la composition des efforts aérodynamiques avec les efforts centrifuges, de remplacer les fatigues de flexion dans la pale par des fatigues de traction.

Soit: $F_{c}$ la force centrifuge s'exercant sur l'élément de pale, $\mathrm{F}_{f}$ la force de flexion aérodynamique, la force résultante $\mathrm{F}_{t}$ donnera une trac-

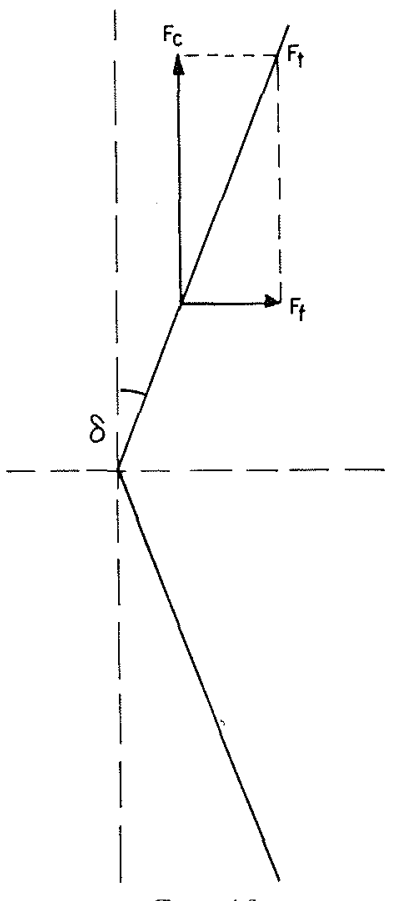

FIG. 16

tion dans la pale si elle est dirigée suivant cette dernière, c'est-à-dire si on a :

$$
\operatorname{tg} \hat{o}=\frac{\mathrm{F}_{f}}{\mathrm{~F}_{c}}
$$

(*) Le rendement propre de la roue baisse rapidement quand $u / v$ augmente. Pour la pale étudiée dans les pages qui précèdent, on peut compter sur les valeurs suivantes: $u / v=5 \quad \eta=85 \%, \quad u / v=10 \quad \eta=70 \%, u / v=15 \quad \eta=55 \%$ 
$\delta$ étant l'angle de la pale avec le plan perpendiculaire à l'axe de rotation.

Cette combinaison ne peut, évidemment, être obtenue que pour une valeur de $\boldsymbol{F}_{f}$, puisque la force centrifuge, comme la vitesse de rotation, est constante. On s'arrange, dans le cas de pales fixes, pour choisir des profils à décrochement aérodynamique, afin d'éviter les variations trop grandes de $\mathbf{F}_{f}$ avec la vitesse du vent. Néanmoins, la difficulté subsiste lors des écarts en direction, car des flexions qui peuvent être importantes apparaissent.

Si la pale est suffisamment flexible, on peut s'arranger pour qu'un accroissement de poussée du vent entraîne un accroissement du dièdre, tel que la résultante des efforts soit toujours sensiblement dans l'axe de la pale. Ceci peut s'obtenir soit par flexion élastique de la pale, soit par rotation autour de son pied à la manière d'une baleine de parapluie, procédé appelé «coning ». On a utilisé et préconisé l'emploi du coning indépendant pour chaque pale. Ce système est loin d'être sans danger, par suite du déséquilibre dynamique générateur de vibrations qu'il fait apparaître dans la roue, précisément à un moment de fatigue accrue. La flexibilité de la pale utilisée pour compenser la flexion par les efforts centrifuges, nécessite un dosage délicat des masses et de l'élasticité. Notons, d'ailleurs, qu'une grande flexibilité fait apparaître un moment de flexion vers l'encastrement de la pale au moyeu. Quelle que soit la disposition adoptée, lorsque la roue est arrêtée face au vent, des efforts de flexion réapparaissent comme dans le cas de la pale perpendiculaire à l'axe de rotation.

Reprenons le calcul des efforts comme dans le cas de la pale perpendiculaire à l'axe de rotation. On suppose que la forme de la pale et les

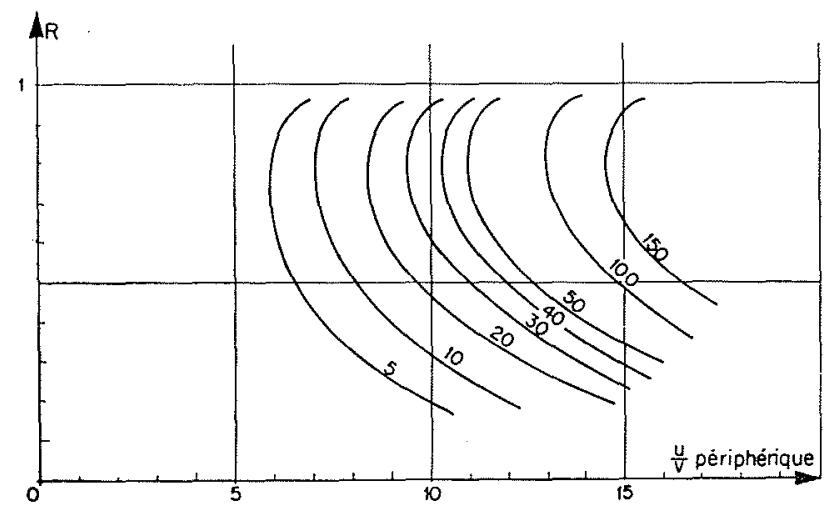

FIG. 17

Fatigue, en $\mathrm{kg} / \mathrm{cm}^{2}$, dans une pale avec dièdre sous l'effet de la force centrifuge. Marche normale face au vent de $12 \mathrm{~m} / \mathrm{s}$. Roue tripale, profil plein, $\delta=10^{\circ}$.

Stress, in $\mathrm{kg} / \mathrm{cm}^{2}$, in a raked blade, due to centrifugal force. Normal operation facing $\alpha$ wind of $12 \mathrm{~m} / \mathrm{s}$. Three-bladed rotor, solid section. $\delta=10^{\circ}$. profils des sections sont identiques, en sorte que les efforts aérodynamiques sont les mêmes. Comme précédemment, les fatigues dues aux forces centrifuges sont indépendantes des dimensions en des points homologues et pour des roues homothétiques. La graphique figure 17 donne la fatigue dans la pale en fonction du rapport $u / v$ périphérique et de la distance à l'axe, le rayon de la pale étant pris pour unité. On s'est placé dans le cas d'une roue tripale avec section pleine et dièdre d'angle $\delta=10^{\circ}$. Pour les petits angles considérés, la fatigue est inversement proportionnelle à l'angle $\delta$. Pour une roue bipale, la fatigue est égale aux deux tiers de la fatigue de la roue tripale.

Les fatigues ainsi obtenues sont beaucoup plus faibles que celles qui sont atteintes dans le cas d'une pale travaillant entièrement à la flexion. Par suite de la forme de la pale, la fatigue maximum est atteinte dans le tiers périphérique de la pale et non pas au pied.

Ainsi que nous l'avons dit plus haut, pour que la force centrifuge puisse, en se composant avec les forces aérodynamiques, supprimer les flexions dans la pale, il faut choisir convenablement la répartition des masses de la pale. La force aérodynamique $\mathrm{F}_{f}$ est imposée par les conditions de marche de la machine, la force centrifuge correspondante pour éliminer la flexion est :

$$
\mathrm{F}_{c}=\frac{\mathrm{F}_{f}}{\operatorname{tg} \delta}
$$

Si on appelle $\mathrm{V}$ le volume d'un élément de pale située à une distance $\mathrm{R}$ de l'axe et se déplaçant avec une vitesse tangentielle $u$, la densité $\gamma$ de cet élément de pale, supposée la même dans toute Ia section, sera :

$$
\gamma=g \cdot \frac{\mathrm{F}_{c} \mathrm{R}}{\mathrm{V} \cdot u^{2}}
$$

On a calculé les valeurs des densités nécessaires pour une roue tripale avec $\delta=10^{\circ}$ dans les conditions de marche précédentes. Les résultats de ce calcul sont portés sur le graphique figure 18 qui met en lumière la nécessité de réaliser des densités faibles. Il sera souvent difficile d'obtenir directement les densités désirées; on peut y arriver par l'emploi de matériaux de densité élevée, mais comportant un grand nombre de trous, de façon à réaliser une structure cellulaire fine, dont la densité moyenne correspond au chiffre désiré. Dans ces conditions, il convient non plus de considérer la fatigue o telle qu'elle a été calculée précédemment (fig. 17), mais le rapport $\sigma / \gamma$ représentant la fatigue ramenée à une densité unité. Ón constate alors que les fatigues réelles sont nettement plus élevées que ne le faisait supposer le simple examen de la figure 17.

Si la force centrifuge permet, grâce au dièdre, 


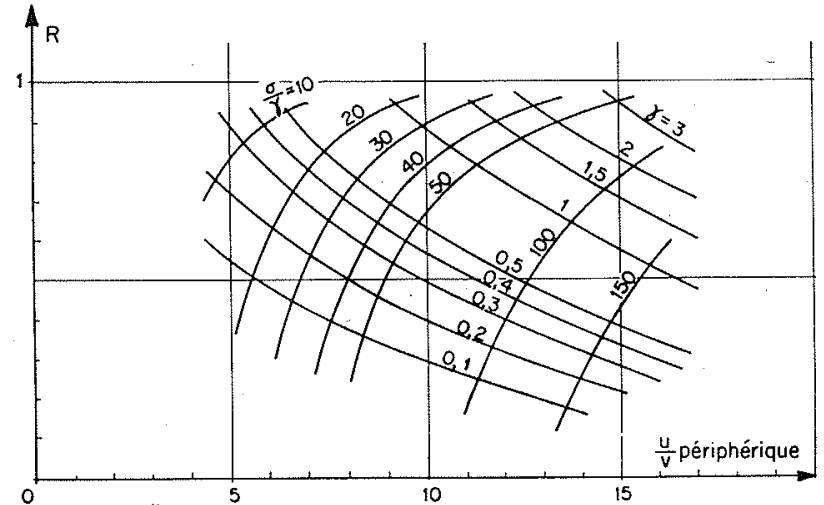

FIG. 18

Densité $\gamma$ dans une pale avec dièdre pour la compensation des flexions par la force centrifuge, et rapport $\sigma / \gamma$ dans le cas de la marche normale face au vent de $v=12 \mathrm{~m} / \mathrm{s}$. Roue tripale, profil plein, $\delta=10^{\circ}$.

Density $\gamma$ in a raked blade for the compensation of the bending by the centrifugal force, and ratio $\sigma / \gamma$ in the case of normal.operation facing a wind of $12 \mathrm{~m} / \mathrm{s}$. Three-bladed rotor, solid section, $\delta=10^{\circ}$.

d'éliminer dans une certaine mesure la flexion en marche normale, il n'en est plus de même lorsque la pale est arrêtée face au vent. Il convient d'examiner à quelle fatigue on est conduit dans ce cas. Les efforts face au vent à l'arrêt, pour le vent maximum de $63 \mathrm{~m} / \mathrm{s}$ qui a été considéré, ont été calculés au paragraphe $a$ ); ils sont évidemment les mêmes ici. Les fatigues pour une section pleine sont également les mêmes mais, compte tenu des faibles densités à réaliser, on envisagera non pas les valeurs de $\sigma$, mais les valeurs de $\sigma / \gamma$ qui deviennent rapidement très importantes. Le graphique figure 19 donne, dans le mode de représentation que nous avons utilisé

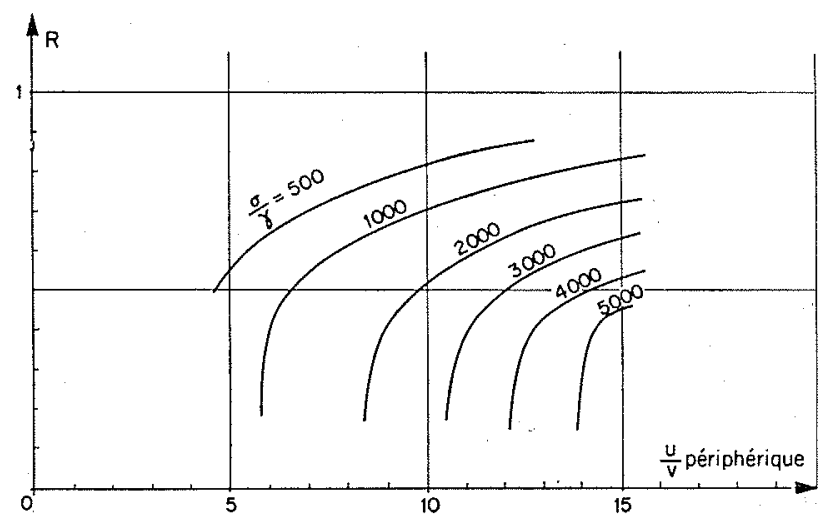

FIG. 19

Rapport fatigue/densité $\sigma / \gamma$ dans une pale avec dièdre arrêtée face au vent par vent de $63 \mathrm{~m} / \mathrm{s}$. Roue tripale, profil plein, $\delta=10^{\circ}$.

Stress/density ratio $\sigma / \gamma$ in a raked blade at standstill facing a wind of $63 \mathrm{~m} / \mathrm{s}$. Three-bladed rotor, solid section, $\delta=10^{\circ}$. jusqu'ici, les variations de $\sigma / \gamma$ en fonction de $u / v$ périphérique pour une roue tripale arrêtée face à un vent de $63 \mathrm{~m} / \mathrm{s}$, la pale étant réalisée en matière cellulaire homogène. Le graphique figure 20 donne des résultats analogues dans le cas où la pale est creuse et réalisée en acier, le métal étant placé à la périphérie du profil et l'épaisseur étant choisie de facon à obtenir la répartition de masse désirée. Ce dernier cas est évidemment plus favorable, car la matière est mieux utilisée pour résister à la flexion. Néanmoins, on constate que les fatigues obtenues pour l'arrêt face au vent sont beaucoup plus élevées que pour une pale n'utilisant pas l'effet centrifuge. En outre, la fatigue maximum est

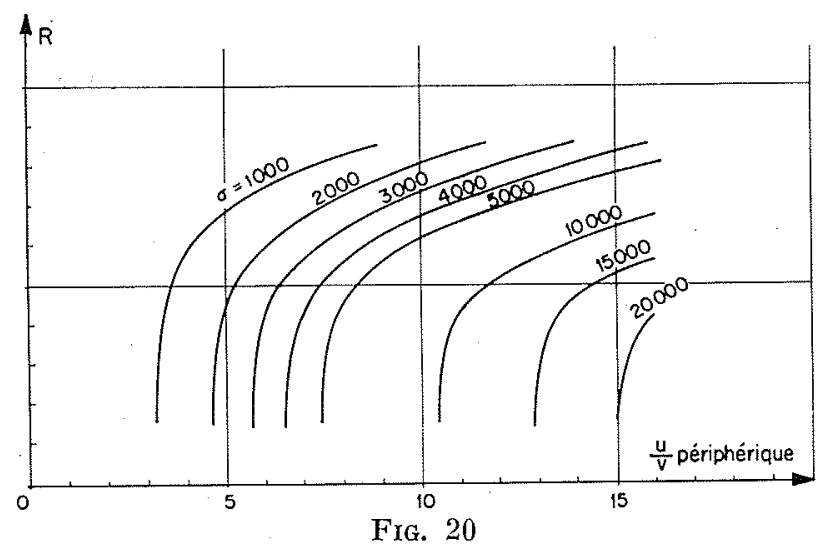

Fatigue, en $\mathrm{kg} / \mathrm{cm}^{2}$, dans une pale avec dièdre arrêtée face au vent par vent de $63 \mathrm{~m} / \mathrm{s}$. Roue tripale, $\delta=10^{\circ}$, profil creux en acier. L'épaisseur varie le long de la pale de telle façon que soit obtenue dans chaque section la masse correspondant à la densité du graphique de la figure 18. Cas de la compensation de la flexion en marche normale par la force centrifuge.

Stress, in $\mathrm{kg} / \mathrm{cm}^{2}$, in a raked blade at standstill facing a wind of $63 \mathrm{~m} / \mathrm{s}$. Three-bladed rotor, $\delta=10^{\circ}$, hollow steel section. The thickness varies along the length of the blade in such a way that in each section the mass corresponding to the density in the graph of Fig. 18 is obtained. Case of the compensation of the bending in normal operation by the centrifugal force.

obtenue au pied de la pale. On peut lever la difficulté en adoptant une répartition de masse le long de la pale, telle que la compensation ne se fasse pas au pied de la pale, où elle est d'ailleurs moins intéressante. Malgré tout, ce sont les efforts machine arrêtée face au vent maximum qui, dans le cas de la roue avec dièdre, limiteront les possibilités de construction.

De cet examen, on peut tirer les conclusions suivantes: la pale perpendiculaire à l'axe de la roue peut raisonnablement, avec des matériaux courants, être utilisée jusqu'à une vitesse limite de l'ordre de $u / v=9$, mais son utilisation économique parait se situer entre 6 et 7 . Pour ce type 
de pale, la fatigue roue arrêtée est faible par rapport à la fatigue en marche normale.

La pale avec dièdre permet d'atteindre des vitesses plus élevées, mais, économiquement, sa vitesse sera limitée par la baisse de rendement. La fatigue roue arrêtée face au vent maximum provoque les fatigues maximums et limitera la vitesse admissible à un rapport $u / v$ de l'ordre de 10 à 11, vitesse qui, d'ailleurs, entraînerait des baisses de rendement exagérées. Le premier type de pale est bien adapté à l'emploi de pales orientables, le deuxième à l'emploi de pales fixes avec décrochage aérodynamique.

On a constaté, par ailleurs, l'intérêt des roues à deux pales par suite de la réduction de fatigue qu'elles entraînent pour une même vitesse de rotation. Mais d'autres éléments que la fatigue doivent être pris en considération et, particulièrement, les vibrations. Lor's de sa rotation, une hélice à deux pales présente une variation cyclique de son moment d'inertie transversal, celui-ci passe successivement par un maximum et par la valeur nulle deux fois par tour. Il en résulte, lors des mouvements d'orientation, une variation cyclique du couple gyroscopique, qui peut être génératrice de vibrations. Un tel phénomène n'existe plus dès que le nombre de pales est égal ou supérieur à 3 .

En outre, lorsqu'une pale tourne dans un vent présentant un gradient vertical, ce qui est généralement le cas, elle est soumise à des effors aérodynamiques subissant une variation cyclique. Il en résulte une variation cyclique des réactions de la roue sur ces appuis et, en particulier, on voit apparaître un couple à variation cyclique ayant tendance à faire osciller la machine autour de son axe d'orientation. Ce couple présente deux maximums et deux minimums par tour de roue pour une hélice bipale; il présente trois maximums et trois minimums par tour pour une hélice tripale. Pour un vent et un gradient donnés, et pour une roue de diamètre et de vitesse données, l'amplitude de la variation de ce couple d'orientation sous l'effet du gradient du vent est dix fois plus grand avec la roue bipale qu'avec la roue tripale.

Si la roue bipale est plus économique de construction et plus rapide que la roue tripale, elle est, par contre, génératrice de vibrations et nécessite des précautions spéciales. Son emploi, de ce fait, n'est pas à conseiller dans une machine déjà par sa nature sujette aux vibrations; aussi ne faut-il pas s'étonner de voir la plupart des réalisations ou des projets établis avec des roues tripales.

\section{Problèmes posés par l'équipement électrique.}

Il ne sera pas question ici des aérogénérateurs fonctionnant isolément, le plus souvent sur batterie d'accumulateurs, mais seulement les machines destinées à débiter sur un réseau alternatif de grande puissance. Indépendamment des sujétions propres au réseau, l'aérogénérateur impose des conditions de marche particulières qui influeront sur le choix du matériel électrique. Ces conditions sont les suivantes : emploi d'un matériel rustique et économique ne nécessitant que le minimum d'entretien, fonctionnement entièrement automatique, variation de puissance fréquente et rapide, accrochages sur le réseau très fréquents, couple moteur subissant des variations cycliques, ensemble du mécanisme soumis à des conditions atmosphériques très dures et à des vibrations importantes.

Quelle que soit la solution électrique adoptée, un réglage mécanique de la puissance s'impose. Ce réglage peut s'effectuer soit par orientation des pales, soit par frein aérodynamique, soit être purement aérodynamique par l'emploi de profils travaillant à la puissance nominale au voisinage de leur point de décollement. La grandeur détectée peut être soit la vitesse, soit la puissance, soit une poussée aérodynamique sur un organe fixe ou tournant. Si le réglage tachymétrique convient pour les aérogénérateurs entraînant des génératrices continues, dans le cas des machines débitant sur un réseau à fréquence constante, l'emploi du réglage « puissance-métrique » est beaucoup plus indiqué. Le réglage mécanique de la puissance de l'éolienne fonctionnera le plus souvent comme limiteur de puissance; il fonctionnera en outre comme limiteur de vitesse lorsque la génératrice sera décrochée du réseau. Il devra done comporter un organe wattmétrique et un organe tachymétrique, ce dernier pouvant se réduire à un simple limiteur centrifuge. Dans le cas d'une machine à pales fixes utilisant des profils à décrochement, il faudra adjoindre à la roue un système de freinage pour éviter l'emballement en cas de décrochage du réseau. La nécessité de ce système de freinage, d'une efficacité toujours aléatoire, fait perdre beaucoup de l'intérêt de la solution à pales fixes. La combinaison la plus heureuse, dans le cas des pales fixes, consiste non pas à absorber le couple par un frein sur l'arbre, mais par un système aérodynamique en bout de pales, dont la sécurité d'emploi est plus grande.

Plusieurs solutions peuvent être adoptées pour la production de courant alternatif et fréquence constante; ces solutions seront classées en trois catégories :

$1^{\circ}$ Groupe intermédiaire entre éolienne et génératrice;

$2^{\circ}$ Génératrice asynchrone entraînée directement par l'éolienne et couplée sur le réseau; 


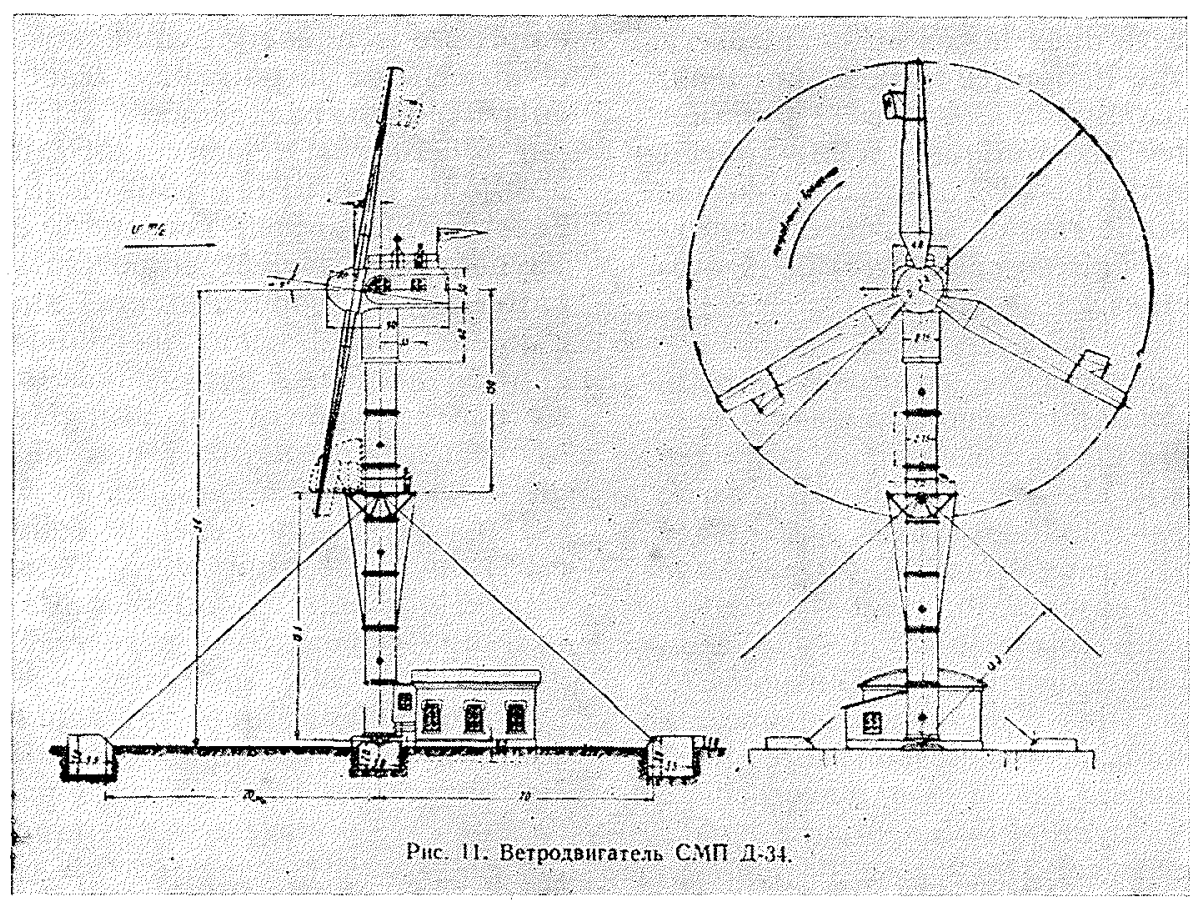

Fig. 21

Aérogénérateur SMP-D-34. Roue de $34 \mathrm{~m}$ de diamètre, système SabininKrasnowski. Par vent de $12 \mathrm{~m} / \mathrm{s}$, cette machine fournit $250 \mathrm{~kW}$ à $33 \mathrm{tr} / \mathrm{mn}$. La génératrice synchrone est placée dans le bâtiment au sol ainsi qu'une génératrice auxiliaire courant continu de $55 \mathrm{~kW}$ utilisée par vent faible pour la charge d'accumulateurs. (Extrait de l'ouvrage de Sidorov.)

SMP-D-34 aerogenerator. Rotor of $34 \mathrm{~m}$ diameter, Sabinin-Krasnowski design. In a wind of $12 \mathrm{~m} / \mathrm{s}$, this machine supplies $250 \mathrm{~kW}$ at $33 \mathrm{r} . \mathrm{p} . \mathrm{m}$. The synchronouls generator is placed in the building on the ground, as well as a $55-\mathrm{kW}$ direct-current auxiliary generator used in low winds for charging accumulators. (Extract from the work of Sidorov.)

$3^{\circ}$ Génératrice synchrone entraînée directement par éolienne et couplée sur le réseau.

Dans la première catégorie seront rangées des solutions très diverses dont voici les plus intéressantes :

a) Entraînement par l'éolienne d'une génératrice de courant continu ne nécessitant pas un réglage très précis de la vitesse de rotation. Le courant continu ainsi produit est ensuite transformé en courant alternatif, soit par un onduleur, soit par une commutatrice. La transformation peut ètre effectuée soit pour chaque aérogénérateur, soit pour un groupe d'aérogénérateurs. Les progrès récents réalisés dans le domaine des génératrices électrostatiques n'excluent pas la possibilité de production de courant continu à haute tension pouvant être transportẻ à distance et transformé alors si nécessaire pour les besoins de l'utilisation.

b) Génératrice synchrone classique couplée au réseau, entraînée par une turbine à air, alimentée elle-même par la dépression créée par un aérogénérateur à pales creuses (solution Andreau). Il est curieux de constater que la grande machine de $100 \mathrm{~kW}$ réalisée suivant ce principe n'utilise pas le principal avantage qu'offre cette disposition, à savoir la possibilité de reporter le réglage sur la turbine à air de petites dimensions et d'utiliser une roue à pales fixes. Au contraire, on a adopté une roue à pales orientables, au prix d'une grande complication mécanique. Dans ces conditions, les seules particularités de la machine sont l'originalité indiscutable et aussi le mauvais rendement.

c) Une disposition plus heureuse consisterait à utiliser comme intermédiaire entre l'hélice et la génératrice une transmission hydraulique réalisée de la manière suivante : une pompe volumétrique à engrenage débitant sur une conduite commune reliant plusieurs éoliennes et alimentant une turbine entrainant l'alternateur classique. Il y aurait dans ce cas intérêt à monter la pompe volumétrique à la place du multiplicateur. Le rendement d'une telle transmission pourrait être de l'ordre de $80 \%$, donc bien supérieur à celui de la transmission à air de l'éolienne à pales creuses. L'emploi de plusieurs éoliennes pour alimenter une même génératrice aurait l'avantage d'assurer une production d'énergie plus régulière nécessitant des manouyres d'accrochage moins 
fréquentes. En outre dans le cas où on utilisera une turbine du type Pelton (ce qui est judicieux, car une telle transmission doit être à pression élevée pour" rester économique), il n'y aurait aucun inconvénient à laisser l'alternateur couplé en permanence sur le réseau, la roue de la turbine tournant dans l'air en cas d'arrêt de la production des éoliennes.

La génératrice asynchrone paraît bien être la solution la plus économique et la plus rustique. Outre sa simplicité de construction et l'absence de contact tournant, elle présente dans le cas de l'entrainement par éolienne deux avantages considérables : la grande facilité de couplage sur le réseau, et l'absence du risque d'oscillation. Il n'y a aucun inconvénient à coupler la machine avec un écart de vitesse de plusieurs \% par rapport à la vitesse de synchronisme, la surpuissance instantanée correspondante étant de très courte durée. La vitesse d'accrochage peut être détectée au moyen d'un organe tachymétrique rudimentaire. "Outre les protections habituelles, l'appareillage se réduit alors à un contact tachymétrique commandant le couplage sur le réseau et à un relais à retour de courant assurant le découplage en cas de baisse de vitesse du vent. Si la génératrice asynchrone ne nécessite pas d'excitatrice, par contre, elle emprunte son énergie magnétisante au réseau et absorbe de la puissance réactive. Cette particularité n'est pas gênante si l'énergie fournie par éolienne est relativement faible par rapport à celle du réseau; elle pose un problème dès que l'énergie fournie par l'ensemble des génératrices asynchrones devient importante. L'emploi de batteries de condensateurs statiques permet d'améliorer le facteur de puissance. On peut, enfin, demander à des groupes tournants, répartis convenablement sur la ligne alimentée par aérogénérateurs asynchrones, de fournir l'énergie magnétisante nécessaire.

Dans le cas où l'énergie éolienne disponible est faible par rapport à l'énergie totale du réseau, une solution intéressante consiste à utiliser une génératrice à cage résistante et grand glissement. Cette génératrice serait entraînée par une éolienne dont le réglage de puissance se ferait simplement par orientation des pales et équilibrage des efforts sur les pales par ressort. Ce mode de réglage très rustique et sans servomoteur se caractérise par un certain écart de vitesse qui, compte tenu de la pente importante de la caractéristique de couple de la génératrice à grand glissement, ne se traduira que par un faible écart relatif de puissance. On réaliserait ainsi une machine dont la limitation de puissance se ferait sans servo-moteur ni régleur.

\section{FIG. 22}

Prix de l'équipement électrique avec génératrice asynchrone protégée à $1.500 \mathrm{tr} / \mathrm{mn}$ et condensateurs de compensation, le prix d'une génératrice asynchrone de $100 \mathrm{~kW}$ étant pris comme unité.

Cost of the electrical equipment with protected asynchronous generator at 1,500 r.p.m. and compensation capacitors, the cost of a $100-\mathrm{kW}$ asynchronous generator being taken as unity

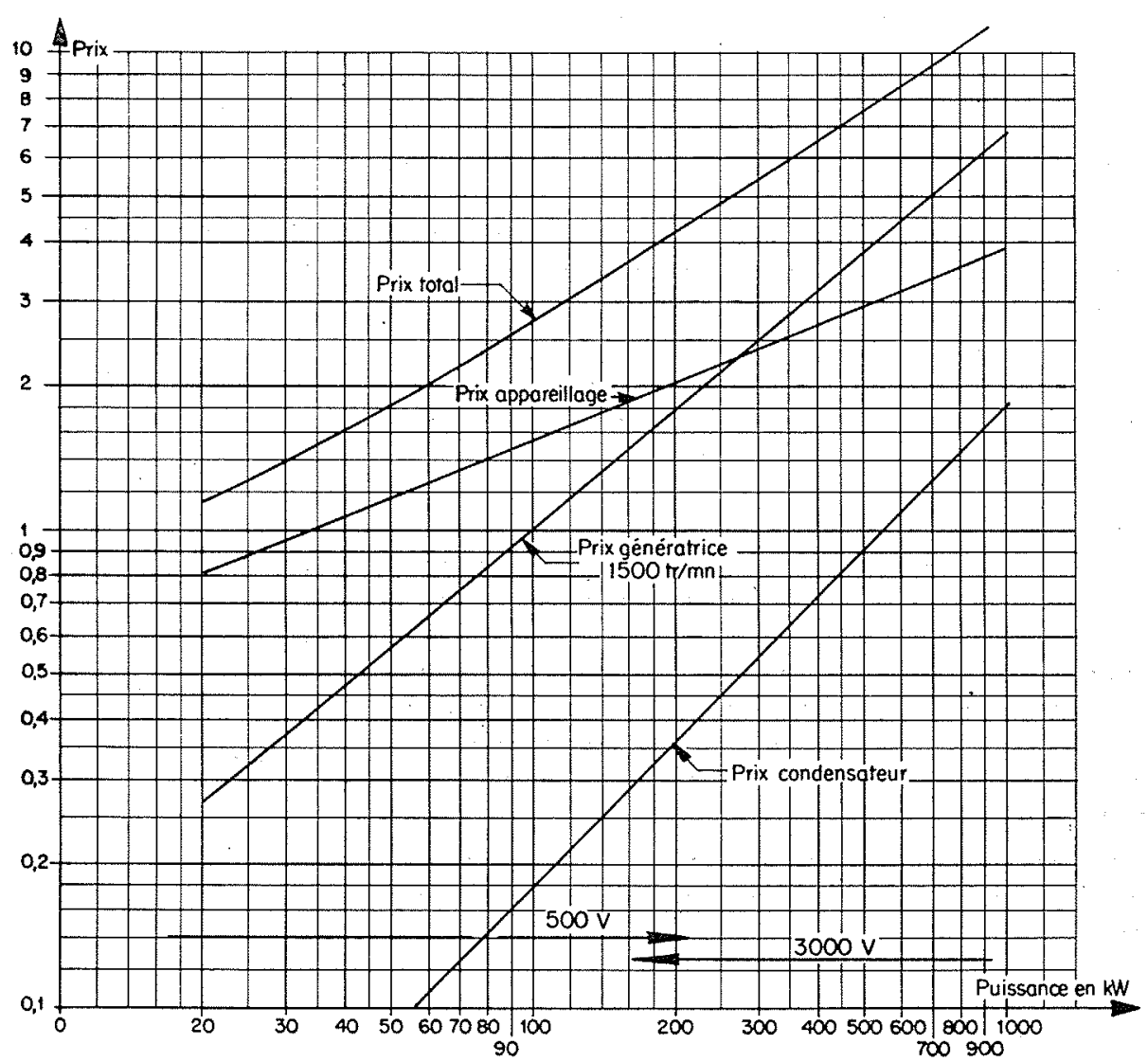


La génératrice synchrone offre le gros avantage de fournir elle-même, par son excitatrice, un courant magnétisant, ce qui semble en favoriser l'emploi pour la production d'une énergie importante d'origine éolienne. Elle présente, toutefois, sous sa forme classique un certain nombre d'inconvénients. La présence de l'exci- tatrice, machine à collecteur, contribue à augmenter le prix du groupe et nécessité une surveillance plus importante que la machine asynchrone qui ne comporte pas de contact tournant. Il est peut-être possible de s'affranchir de cette difficulté par l'emploi d'alternateurs autoexcités, dont les inducteurs sont alimentés par

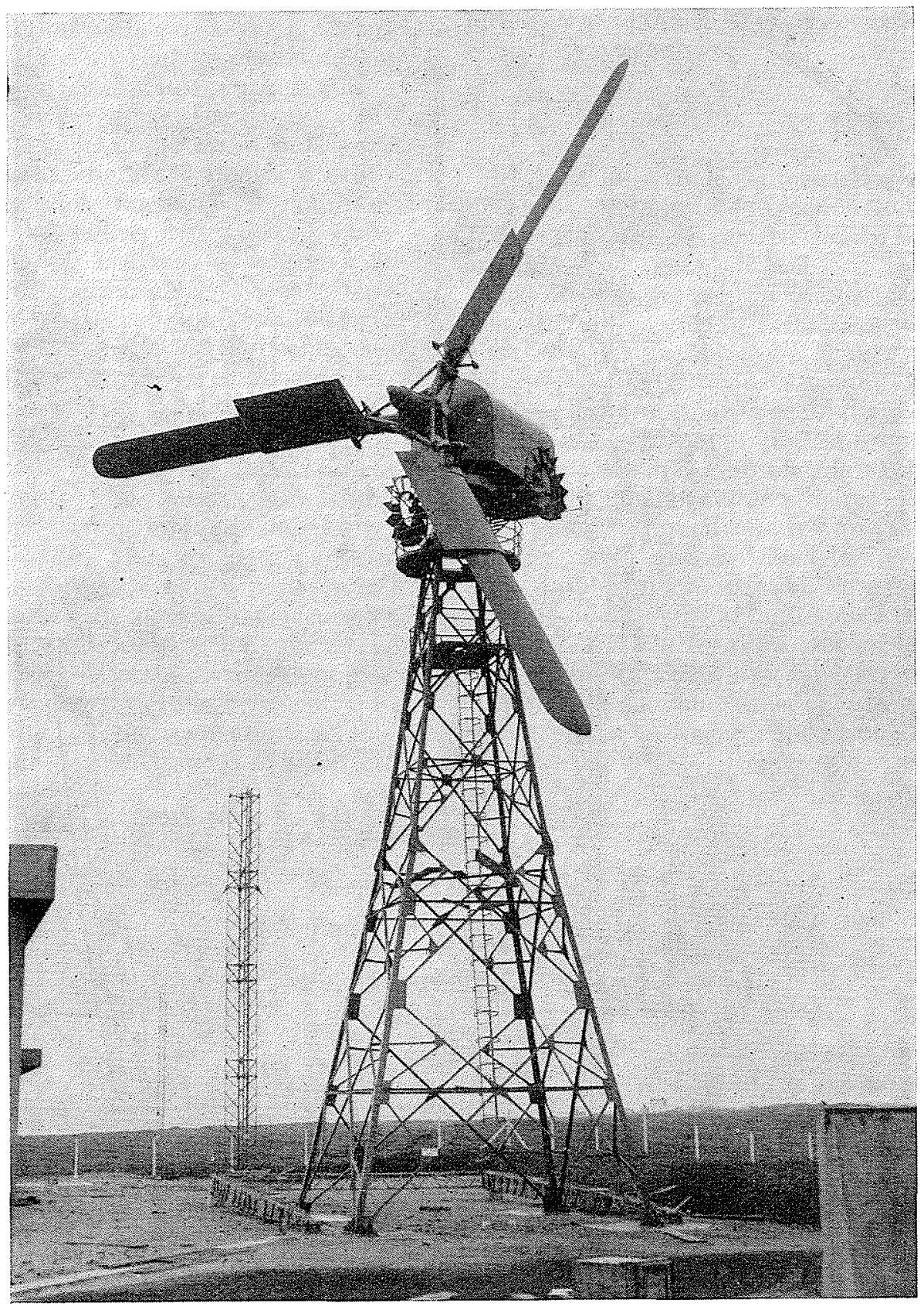

FIG. 23

Aérogénérateur NEYRPIC D-21. Roue de $21 \mathrm{~m}$ de diamètre à pales orientables. Par vent de $11 \mathrm{~m} / \mathrm{s}$, cette machine fournit $132 \mathrm{~kW}$ à $56 \mathrm{tr} / \mathrm{mn}$. La génératrice est du type asynchrone.

Neyrpic D-21 aerogenerator. Rotor of $21 \mathrm{~m}$ diameter with variable-pitch vlades. In a wind of $11 \mathrm{~m} / \mathrm{s}$, this machine supplies $132 \mathrm{~kW}$ at 56 r.p.m. The generator is of the asynchronous type. 


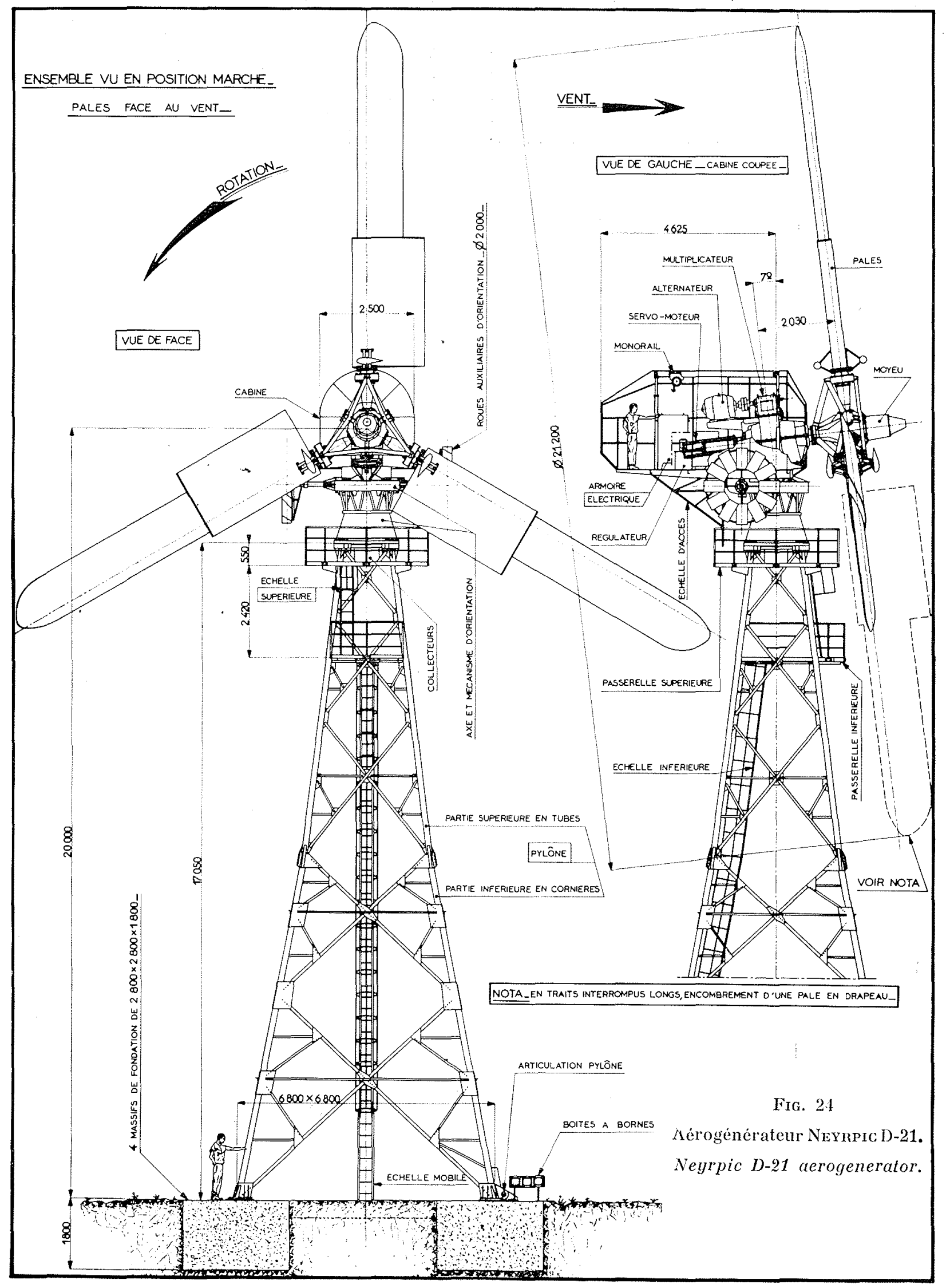


un enroulement spécial du stator et un redresseur.

Alors que, dans la génératrice asynchrone, la puissance électrique déversée sur le réseau est automatiquement ajustée à la puissance mécanique disponible sur l'arbre par le simple jeu du glissement, dans la génératrice synchrone, par contre, il faut ajuster la valeur du courant d'excitation en fonction de la puissance à échanger, d'où la nécessité d'un réglage électrique de l'excitation si on veut maintenir au $\cos \varphi$ une valeur convenable. Ce réglage électrique vient s'ajouter au réglage mécanique de la puissance de l'éolienne. On peut, toutefois, mettre à profit les caractéristiques des machines synchrones dans un ensemble d'aérogénérateurs dont les uns seraient équipés de génératrices synchrones et les autres de génératrices asynchrones. Dans une telle combinaison, les besoins en énergie magnétisante des génératrices asynchrones seraient satisfaits par les génératrices synchrones, convenablement surexcitées.

L'accrochage de la génératrice synchrone sur le réseau demande un ajustage de la vitesse de rotation beaucoup plus précis que la machine asynchrone. La fréquence de cette opération pour les aérogénérateurs fait, de cette particularité, une sérieuse difficulté. On a pensé tourner cette difficulté en interposant un accouplement formant roue libre entre l'éolienne et la génératrice, et en laissant cette dernière couplée en permanence au réseau. Ce n'est, en fait, que déplacer la difficulté, car la réalisation de tels accouplements, pour des puissances élevées, est loin d'être économique.
Une solution plus intéressante consiste à prévoir sur le rotor de la génératrice des amortisseurs importants, permettant la marche en asynchrone lors du couplage. La présence de tels amortisseurs est d'ailleurs souhaitable, en raison du risque d'oscillation propre aux génératrices synchrones. On sait, en effet, que ces machines possèdent une période propre d'oscillation dépendant de leur moment d'inertie et du couple synchronisant.

$$
\mathrm{T}=\frac{0,25}{p} \sqrt{\frac{\mathrm{PD}^{2} \cdot f}{\left(\mathrm{I}_{k} / \mathrm{I}\right) \mathrm{N}}}
$$

T étant la période (en secondes), $p$ le nombre de paires de pôles, $f$ la fréquence, $\mathrm{PD}^{2}$ en $\mathrm{kg} . \mathrm{m}^{2}$, $\mathrm{I}_{k} / \mathrm{I}$ le rapport de court-circuit, $\mathrm{N}$ la puissance en $\mathrm{kVA}$.

La forme même de cette période propre d'oscillation montre qu'elle dépend de l'état de charge de la machine; elle couvre donc une certaine plage, ce qui constitue une difficulté pour éviter que la période d'oscillation de la génératrice, ou ces harmoniques, ne coïncident avec les périodes d'excitation de l'éolienne (passage des pales devant le pylône, etc.).

Ce rapide examen montre que si l'équipement d'une grande énergie d'origine éolienne nécessite l'emploi, même partiel, de génératrices synchrones, celles-ci, par suite de leur's conditions particulières d'utilisation, seront d'une conception assez différente des génératrices classiques. Le choix de ces machines et un éventuel dosage synchrone-asynchrone dépendront de l'étude du réseau.

(A suivre.) 


\title{
The generation of electricity by windmills
}

\author{
ISY L. VADOT \\ CONSUltiNg ENGINEER AT ETAIHISSEMENTS NEYMPIC \\ (Translation by Madge E. THOMPSON) \\ Texte français, p. 503 \\ See French text for illustrations
}

\begin{abstract}
Two fundamental problems of windmill design are considered in this article: determining the dimensions of the machine and its output. Economic and engineering considerations make it possible to define the most advantageous characteristics for a windmill. It would seem that, in view of available wind power in the Atlantic region, it is of immediate interest to use wind generators.
\end{abstract}

There are two essential features in the problem of utilizing the energy in the wind. One is its wide dispersion in space, the other its great and unpredictable irregularity. It is a form of energy which, although apparently gratuitous, cannot be made economically attractive unless it is exploited by a suitable design of machine. A few figures will serve as an example.

In a wind speed of $10 \mathrm{~m} / \mathrm{s}$, the maximum power that can be obtained, with a perfect machine, is of the order of $0.3 \mathrm{~kW}$ per $\mathrm{m}^{2}$. In practice, however, the imperfections of the machine make it impossible to obtain more than $0.2 \mathrm{~kW}$ per $\mathrm{m}^{2}$. There is no site at which such power is regularly available, for the wind speed changes continuously; from a negligible value, in a calm period, it may, in a storm, rise to $50 \mathrm{~m} / \mathrm{s}$ and even more, which would give a power 125 times greater than that envisaged here, i.e., $25 \mathrm{~kW}$. per $\mathrm{m}^{2}$.

The irregularity in time is such that the number of hours for which a certain power is available diminishes rapidly when this power increases, so that it is quite out of the question to build a machine capable of supplying $25 \mathrm{~kW}$ per $\mathrm{m}^{2}$. Economic considerations lead to the choice of a much lower figure-something around 0.2 or $0.3 \mathrm{~kW}$ per $\mathrm{m}^{2}$ for a moderately windy site. The machine must, however, be able to stand up to very strong winds.

The first need is to design a machine which will be economic in spite of the enormous difference between the power that can be captured and the maximum power that may traverse it. We showed in a previous article " A Synoptic Study of the Different Types of Windmills", la Houille Blanche, March-April, 1957) the principal features which enable a machine to operate under large variations in wind speed. The necessity for adapting the consumption of the energy to the irregularity, in time, of its production is another important consideration.

Several attempts have been made to remedy the irregularity of wind energy by storing it for use at a suitable time and making the consumption independent of the production. Nearly all the known means have been tried or studied : pumped storage, kinetic storage in a high-speed fly-wheel, thermal storage by the direct production of heat in a Froude type or brake, electrical storage by battery, chemical storage through the electrolytic production of hydrogen and oxygen, etc. Compensation is another method; in this the windmill works in parallel with another 
source of energy, the production from which is complementary to the production from the windmill. A third method is to choose a type of consumption which can conveniently be adjusted ot the irregularities of production; this was, in fact, the case with corn grinding at the time when windmills merited their name.

Apart from certain special cases, the irregularity of wind energy is adapted to two important functions: water pumping and the generation of electricity. The question of pumping was discussed in a previous article "Water Pumping by Windmills ", la Houille Blanche, September, 1957) and this article will deal with the different problems raised by the construction of wind machines for the generation of electricity.

The conditions of operation of an aerogenerator can certainly be very diverse, but, for the sake of simplicity, it will be convenient to consider two methods of use. In one, the aerogenerator works independently; in the other, it works in parallel with a source of electrical energy which is assumed to be of sufficient power to meet, if the occasion arises, the whole of the demand for power.

The independant aerogenerator must obviously function with storage. Except when the energy is to be used only for heating, or when there is a possibility of using pumped storage close at hand, such a machine will be equipped to produce direct current to charge a battery. It will generally be of a rather low power, so that its dimensions will be decided by the production required and not by considerations of mechanical construction. The economic conditions in this case are rather special, the cost of the energy very often being a less important consideration than the possibility of making it available. From the mechanical point of view, since the drive of a direct current generator does not require that the speed of rotation should be rigorously constant, a very simple system of control is adequate and, for low powers, may merely consist of turning the rotor out of wind. For medium powers, variable-pitch blades with spring control are very satisfactory. The rotor will naturally be of the high-speed type to facilitate the drive of the generator. A safety device will be necessary to avoid racing if a fault should occur on the electrical side. Briefly, in general principles, the independant aerogenerator will be very similar to a windmill used for driving a centrifugal pump.

The conditions for an installation designed to work in parallel with another source of electrical energy, particularly to feed into a large network, are quite different. The dimensions to be given to the machine, and the choice and arrangement of the parts, will then be determined by mechanical and economic considerations. The power capacity of each generating unit will make it necessary to examine many points which can be neglected for small machines.

\section{TECHNICAL PROBLEMS}

\section{Fundamental relation between power, diameter and wind speed}

When a stream of air passes through a given vertical area, the total flow of energy is entirely kinetic; neither pressure nor temperature affect it, since these quantities are constant in a stream of air moving uniformly. For a stream with a vertical cross section of $1 \mathrm{~m}^{2}$, at a speed of $v \mathrm{~m} / \mathrm{s}$, the total power, expressed in $\mathrm{kW}$, is :

$$
\mathrm{N}_{t}=0.000613 v^{3}
$$

for air at $15^{\circ} \mathrm{C}$ and a pressure of $760 \mathrm{~mm}$ of mercury, i.e., of density $1.23 \mathrm{~kg} / \mathrm{m}^{3}$.

Not all this power can le captured. A certain quantity of kinetic energy remains in the air which has passed through the rotor; this kinetic energy cannot be zero, since it is necessary to evacuate the air which has done its work, but it cannot be captured. If $v$ is the speed up-wind of the rotor and $v_{2}$ the speed down-wind, it can be shown that, for a given power available, that which can be captured depends on the ratio $v_{2} / v$ and is maximum when $v_{2} / v=1 / 3$. Under these conditions, the power that can be captured is equal to $16 / 27$ of the total power. Betz establised this theory in 1927.

In a stream of vertical cross section $1 \mathrm{~m}^{2}$, the maximum power that can be captured, according to Betz' theory, is expressed as :

$$
\mathrm{N}_{\mathrm{B}}=16 / 27 \times 0.000613 v^{3}
$$

or :

$$
\mathrm{N}_{\mathrm{B}}=0.000364 v^{3}
$$

the power being expressed in $\mathrm{kW}$ and the speed in $\mathrm{m} / \mathrm{s}$. In practice a machine does not capture all this power; its different losses have to be taken into account-mechanical losses, losses through friction on the blades, losses through marginal eddies, etc. If $\eta$ is the efficiency (in relation to Betz' ratio) of the machine, the power 
that can be effectively captured on the shaft of the rotor is :

$$
\mathrm{N}=\eta \cdot \mathrm{N}_{\mathrm{B}}=\eta(16 / 27) \mathrm{N}_{t}
$$

It is customary to combine the efficiency $\eta$ and Betz' ratio in one term, which is called the power coefficient $\mathrm{C}_{\mathrm{N}}$, where :

$$
\mathrm{C}_{\mathrm{N}}=\mathrm{N} / \mathrm{N}_{t}
$$

This expression is sometimes called the efficiency, which leads to confusion, since it is derived from a power which cannot be captured.

For a rotor of diameter $\mathrm{D}$, the power obtained is :

$$
\begin{gathered}
\mathrm{N}=\frac{\pi \mathrm{D}^{2}}{4} \eta \frac{16}{27} 0.000613 v^{3} \\
=0.000286 \eta \mathrm{D}^{2} v^{3}
\end{gathered}
$$

or, expressed in terms of the $\triangleleft$ power co-efficient,

$$
\mathrm{N}=0.000482 \mathrm{C}_{\mathrm{N}} \mathrm{D}^{2} v^{3}
$$

the power being expressed in $\mathrm{kW}$, the diameter in metres and the speed in $\mathrm{m} / \mathrm{s}$.

The type of rotor is generally characterized by its tip-speed ratio, i.e., the ratio of the tipspeed $u$ to the wind speed $v$. The speed of rotation $n$, in r.p.m., is obviously related to the tipspeed ratio by the expression :

$$
n=\left(\frac{u}{v}\right) \frac{60}{\pi} \frac{v}{\mathrm{D}}
$$

On the basis of the preceding formulae a diagram has been drawn up to show, as a function of the wind speed and of the diameter of the rotor, the power that can be captured on the shaft, and the speed of rotation, for different values of $u / v$, with a good average value of the efficiency, $\eta=70 \%$, i.e., $\mathrm{C}_{\mathrm{x}}=0.415$, and with air at $15^{\circ} \mathrm{C}$ and a pressure of $760 \mathrm{~mm}$ of mercury. This diagram gives, with a fair approximation, the dimensions to be taken in designing a machine.

\section{Note on the comparison between a windmill and a helicopter}

The low value of the efficiency of an aerogenerator, in relation to the total power of the stream of air which passes through it, may seem surprising, but it is characteristic of every machine which cannot capture the kinetic energy as it passes out. A striking example is given by the comparison between the windmill and the helicopter. These two machines, one driving, the other driven, have several points in common and a certain similarity in the aspect if their rotors. Taking the characteristics of a helicopter of recent design, we can compare its efficiency with that of an aerogenerator having a rotor of the same diameter.

Consider the Westland-Wessex helicopter which has just been put in service in the Royal Navy and is derived from the classic Sikorsky S-58 with the piston engine replaced by a Napier turbine. It has the following characteristics :

Number of blades on the rotor .... 4

Diameter of the rotor........ $17 \mathrm{~m}$

Speed of rotation........... 221 r.p.m.

Driving power............. 1,070 kW

Total weight............ 5,700 kg

Maximum lifting speed........ $8 \mathrm{~m} / \mathrm{s}$

Under these conditions, the average speed of the stream of air blown by the rotor is $18.6 \mathrm{~m} / \mathrm{s}$. The corresponding tip-speed ratio $u / v$ is 10.7 , a usual value for a helicopter but one which would be rather high for a windmill where extreme ligthness is not necessary.

Evaluating, then, the total power contained in the stream of air, we find $\mathrm{N}_{t}=910 \mathrm{~kW}$. The efficiency of the machine, in relation to this power, is $\eta=85 \%$, which is a high value. But by no means all this power is used. In fact, if we calculate the power corresponding to the ascent of the machine at its maximum lifting speed, we find the effectively used power to be $\mathrm{N}=456 \mathrm{~kW}$. And the true efficiency of the machine is $\eta=456 / 1,070=43.5 \%$. A large part of the power of the engine produces the kinetic energy of the stream blown downwards and this cannot be captured.

Suppose, now, that in the same stream of air, at $18.6 \mathrm{~m} / \mathrm{s}$, a windmill rotor of $17 \mathrm{~m}$ diameter is made to turn. With the figures shown above, an overall efficiency of $41.5 \%$ can be counted on, which will give a power of $375 \mathrm{~kW}$. There is certainly a considerable difference between the $1,070 \mathrm{~kW}$ of the helicopter engine and the $375 \mathrm{~kW}$ which can be captured on the shaft of the windmill, but the two machines have very similar efficiencies, $43.5 \%$ and $41.5 \%$ respectively, for the losses are the same in the two cases.

\section{Atmospheric turbulence}

In making calculations for the different parts of a windmill a serious difficulty is encountered. Although the normal operating conditions, and particularly those which correspond to the rated characteristics, are well defined, the accidental operating conditions, in the course of which nearly all the greatest stresses occur, are very 
imperfectly known. Generally, the maximum value of the wind speed that the machine will be called upon to withstand is defined. Apart from the arbitrary nature of the choice of this maximum value, it is obvious that the problem is, in fact, much more complicated. Not only is the maximum value of the wind speed important, but also the rapidity of the variations, their frequency and amplitude, as well as the magnitude of the changes of direction which accompany those of speed. To approach the problem more closely, it would be necessary to take into account the instantaneous fluctuations in the characteristics of the wind at each point of the area swept by the rotor and to determine the way the rotor integrates the variable stresses at each point.

It is almost impossible to find a perfect solution to such a problem. The most that one can hope to do is to define operating conditions representing approximately the limits within which the accidental stresses are likely to develop. Not the least of the difficulties is that, with a few rare exceptions, anemometer measurements have been made under very different conditions from those in which a windmill operates and generally for rather different meteorological purposes such as the study of the fine turbulence of the atmosphere. Usually the information is supplied by recording anemometers. Apart from the fact that these measure the wind speed only at a point, the recording speeds are such that it is difficult to use them to gain any knowledge of the rapidly varying phenomena which affect windmills. Consequently, in spite of the existence of a considerable number of wind records, very few of them can be used.

As these inadequate measurements make it impossible to take into account the non-uniform winds in the area swept by the rotor, we will assume that we are dealing with a uniform average wind, i.e., a wind having, at any given instant, the same characteristies at every point of the swept area. We will simply define the values representing the variations of this wind, viz, the maximum intensity, the amplitude and frequency of the oscillations round the average value, the acceleration, the duration of gusts and changes in direction.

The maximum wind speed to be considered obviously depends on the district in which it occurs. It can be deduced from the analysis of the anemometer records for a sufficiently long period stretching over several years. Such a maximum speed always takes the form of a peak of very short duration. It is an oscillation of turbulence, of great amplitude, which is superposed on an average wind of distinctly lower value. The very short duration of the maximum recorded peak wind speed causes it to be taken into account, in a particular way, in the calculations. For European districts where the exploitation of wind energy is of interest, and principally for the Atlantic zone, a maximum speed of $55 \mathrm{~m} / \mathrm{s}$, measured at $10 \mathrm{~m}$ above the ground, can be adopted.

According to the nature of the ground surrounding the point of observation or, more exactly, its ruggedness, so will the turbulence extend to a greater or lesser distance. The record of this turbulence gives a very tortuous curve with a succession of alternate maxima and minima. Such a record is characterized fairly well in the following way. By counting, first the minima, and then the maxima, and determining the number of each belonging to a given range of wind speed, we can trace two curves giving respectively the probability of occurrence of the minima and the maxima reaching a certain value of the speed. Thus we obtain two bell-shaped curves, situated on either side of the average value of the speed. These curves are more or less spread out according to the intensity of the turbulence. To each value of the probability $p$, there is a corresponding value of the variation of speed $\Delta v$ or amplitude of the turbulence. This amplitude increases when the probability diminishes We may adopt, as the amplitude of the turbulence, that which corresponds, for example, to a probability of $5 \%$ and characterize the turbulence of the wind by the ratio $\Delta v / v_{m}$, that is, the ratio of the amplitude just defined to the average value of the speed. This ratio depends on the place chosen; generally it varies between 0.6 and 1 for measurements made with the standard anemometers used for meteorological purposes. It is certain that the wind speed record depends in detail on the dimensions of the measuring apparatus and on its time of response (i.e., on the scale of the turbulence observed), but it is unlikely that the extreme values used to define $\Delta v$ vary very much.

Atmospheric turbulence can be considered as due to the superposition of eddies on a uniform motion of the wind stream. A simple eddy, driven by the current at speed $v_{m}$, will give rise to oscillations of both speed and direction. Now, although the anemometer records readily give indications of the oscillations of speed, there is generally no indication of the oscillations of direction. These will be evaluated by the following rule of composition of the speeds. It is agreed that an oscillation of speed $\Delta v$ is obtained by the superposition, on a current $v_{m}$, of an eddy of uniform tangential speed equal to $\Delta v / 2$. Under these conditions, when the tangential speed of the eddy is parallel to and in the same direction as $v_{m}$, the maximum speed $v_{m}+(\Delta v / 2)$ 
will be obtained, and when it is in the direction contrary to $v_{m}$, the minimum speed $v_{m}-(\Delta v / 2)$ will be obtained. For all the other orientations of the tangential speed of the eddy in relation to the average speed $v_{m}$, the resultant speed will be obtained from the figure. This, in particular, enables the maximum variation in direction to be determined (Fig. 5) :

Let $\mathrm{AB}=v_{m}-(\Delta v / 2)$ and $\mathrm{AC}=v_{m}+(\Delta v / 2)$; from the point $A$, the tangents are drawn to the circle of diameter $\mathrm{BC}=\Delta v$. Let $\mathrm{AD}$ and $\mathrm{AE}$ be these tangents; they represent the maximum variations in direction. The angle $\beta$, measuring this variation, has a value :

$$
\sin \beta=\frac{\Delta v}{2 v_{m}}
$$

It is the maximum variation in direction corresponding to an average speed $v_{m}$ subjected to an oscillation of amplitude $\Delta v$.

Observations agree fairly well with the results deduced from this rule.

The following are a few readings taken at La Banne d'Ordanche:

\begin{tabular}{|l|c|c|c|}
\hline $\begin{array}{c}\text { MAXIMUM } \\
\text { SPEED }\end{array}$ & $\begin{array}{c}\text { MINIMUM } \\
\text { SPEED }\end{array}$ & $\begin{array}{c}\text { VARIATION } \\
\text { OBSERVED }\end{array}$ & $\begin{array}{c}\text { VARIATION } \\
\text { CaLCULATED }\end{array}$ \\
\hline $53 \mathrm{~m} / \mathrm{s}$ & $39 \mathrm{~m} / \mathrm{s}$ & $\pm 8^{\circ}$ & $\pm 9^{\circ}$ \\
36.5 & 19.7 & $\pm 22.5^{\circ}$ & $\pm 24^{\circ}$ \\
20.5 & 11 & $\pm 17.8^{\circ}$ & $\pm 19^{\circ}$ \\
\hline
\end{tabular}

It is notable that the variations in direction occur principally in a horizontal plane; these are variations in bearing. The variations in a vertical plane are much less (about one-seventh to one-tenth) and are practically negligible when the ground is even and horizontal. Obviously, in the wake of an obstacle the conditions are not the same, but such a location is always to be avoided.

A knowledge of the variations in direction is of the greatest use in designing a machine. In fact, considering the admissible gyroscopic moments, it is not possible to choose a speed of yawing high enough to follow the variations in direction; thus the largest stresses occur in the blades when, as a result of a change of wind, the axis of the rotor makes a certain angle with the direction of the wind. These stresses, varying and changing sign in each rotation of the rotor, subject the blade to dangerous alternating fatigues. These are the conditions which govern the choice of the structure of the blade and not the fatigues which occur in normal operation.
It does not seem that, on the scale of phenomena with which aerogenerators are concerned, an acceleration of the wind greater than $4 \mathrm{~m} / \mathrm{s}$ is encountered. Taking this figure as a basis, we obtain the minimum duration of increases of speed; this increases when the average wind speed increases. Now, as the maximum variations in direction occur in the time taken to pass through the variation of speed $\Delta v$, it is deduced that the variations of direction are slower in a strong wind than in a low wind, a fact which is confirmed by experience.

The vertical wind gradient plays an important part in the calculation of the stresses as well as in the choice of the optimum dimensions to be given to machines. It influences :

- The distribution of the usable energy in the area swept by the rotor;

- The cyclic modification of the stresses on the blades;

-- Displacement of the centre of thrust;

- The aerodynamic efficiency of the cyclic variations of incidence of the relative current;

- The variation of the weight and cost of machines per $\mathrm{kWh}$ produced, as a function of the dimensions of the rotor.

The vertical wind gradient depends partly on the local relief at the point considered and partly on the ruggedness of the regions over which the wind has blown. It is known that at the top of a rounded hill there is a local increase of speed which may have the effect of inverting the usual gradient, so that, contrary to the accepted idea, the speed may be greater near the ground than at a certain height. Apart from this peculiarity, in general, on flat country, the speed variation $v$, as a function of the height $h$ above ground, may be represented by a law of the form :

$$
v=v_{0}\left(h / h_{0}\right)^{1 / n}
$$

$v_{0}$ being the speed at the height $h_{0}$ and $n$ having a value which varies appreciably according to the ruggedness of the terrain over which the wind blows. A typical example of this influence is given by the following fact. When the measurement of vertical distribution is made on the sea coast, the result differs widely according to whether the wind comes from the sea or from the land. For the sea wind, the variation of speed with altitude is very slight, which means that $n$ is large, whereas, for the land wind, the gradient is large and $n$ is smaller. For flat ground with normal ruggedness, $n$ does not fall below 5 . In the study which follows, the value $n=5$ has been taken a value which is favourable to the adoption of machines of large dimensions. 
A very important requirement for the calculation of the resistance of aerogenerators is a knowledge of the curve giving, for high speeds (near the maximum), the duration of each speed. The usual velocity-duration curve, drawn up for calculations of energy, is difficult to use in this case, first because it is not generally precise enough at high winds, its scale of time being too restricted, and secondly because it is based on average speeds and does not take turbulence into account. Suppose that such a curve were drawn taking turbulence into account, it would have the shape shown in Fig. 6. The peak, or highest maximum speed, is the maximum variation obtained from an average maximum wind. The durations, at first very short, increase rapidly when the zone of average speeds is reached. The scale of the abscissae can be graduated in numbers of turns of the rotor, so that the curve gives, for each wind speed, the number of alternations of stresses to which the rotor is subjected. It is useful to put alongside this curve, the curve giving, for the material of which te rotor is made, the variation of the admissible stress as a function of the number of alternations of the stresses. The two curves have approximately the same shape. This remarkable peculiarity makes it possible to use, for the calculation of the stresses at the highest maximum wind, admissible stresses distinctly higher than for the average winds. The comparison of the curves of wind speed and of admissible stresses, as a function of the number of alternations, enables one to determine what wind speed leads to the maximum sections; it is not necessarily the highest maximum wind. In any case, the similarity between the two curves shows that there is no great difference between the sections obtained in designing the machine for the highest maximum wind and those obtained in designing it for an average wind. This is obviously a very favourable cireumstance.

\section{Choice of the rated wind speed}

The wind energy available at a given place is represented in the most significant way by the velocity-duration curve giving, for each value of the wind speed, the number of hours per year during which there is a wind available of a speed which is at least equal to the value considered. For most of the districts where anemometer readings have enabled such curves to be drawn, it is seen that the shape of the velocity-duration curves is the same. If, for all the curves considered, a scale of ordinates is chosen such that the points corresponding, for example, to the duration 4,000 hours per year coincide, the different curves are seen to be nearly identical.
With a change of the scale of the ordinates, it can therefore, as a first approximation, be agreed that a single curve can represent different sites. The change of scale will be simply defined by the value of the average wind in the year, which is also the value of the wind for a duration of 4,000 hours. The velocity-duration curve enables a curve to be drawn giving the power available as a function of the number of hours. The area situated under the power-duration curve (obtained by cubing the ordinates of the velocity-duration curve) will indicate the energy available in the year. But the aerogenerator cannot give all the energy thus theoretically available in the wind because limitations are imposed by other considerations.

When the wind speed increases, the machine begins to supply power only at a certain speed called the "cut-in speed", a speed such that the power available on the shaft of the machine corresponds to the power absorbed by the no-load losses of the whole set (generating losses, gearing losses, losses through the auxiliaries). Then, as the wind speed increases, it attains a certain value called the "rated wind speed", which is the speed at which the machine supplies its rated power. For any value of speed above the rated speed, the power will be kept constant by the regulating system. Finally, if the wind speed continues to increase, it reaches a value called the "furling speed", beyond which the machine is stopped, for reasons of safety, and gives no more power.

The area corresponding to the energy actually given out by the machine in the year will therefore be limited :

(1) at the lower part, by a horizontal line through the ordinate equal to the power corresponding to the "cut-in" wind speed;

(2) on the right, by the power-duration curve;

(3) at the upper part, by a horizontal line through the ordinate equal to the rated power;

(4) on the left, by a vertical line of which the abscissa corresponds to the duration of winds of "furling speed".

This last has practically no influence on the choice of the characteristics of the machine.

The essential value to be determined is the rated wind speed, or the rated power. As to the "cut-in" speed and the no-load losses, they depend on the value of the rated power.

In the calculations used for the determination of the curves in Fig. 8, the total power absorbed by the no-load losses of the set was taken as $10 \%$ of the rated power. Also the time for which the machine is stopped in high winds was 
taken to be uniformly 250 hours in the year.

Under these conditions, it is seen that for a given average wind speed in the year (i.e., for a certain velocity-duration curve characterizing a site) the curve representing the variations of the energy produced in the year as a function of the value of the rated wind speed (i.e., as a function of the rated power of the machine) at first rises, then passes through a maximum and finally falls again as the rated wind speed continues to increase. There is, therefore, for each value of the average wind speed, a value of the rated wind speed which gives the maximum energy possible. This maximum is obtained when :

$$
\begin{gathered}
\text { rated wind speed } \\
=1,9 \times \text { annual average wind speed. }
\end{gathered}
$$

The existence of the maximum is due to the fact that the no-load losses of the set increase when the rated power increases, while the number of hours of operation diminishes.

From the economic point of view, a rated power should be chosen for the aerogenerator corresponding to a lower rated wind speed than that which has just been indicated, because what is more important than any other consideration is to achieve the lowest cost per $\mathrm{kWh}$ of energy produced. Now, in the total cost of the windmill equipment, one part of the charges increases with the capacity installed. As a result of this increase, the economic optimum will be attained for a lower rated wind speed than 1.9 times the annual average wind speed. Finally, the value of the rated wind speed will depend on the relative importance, as part of the total cost of the equipment, of the charges which increase with the installed capacity. As a good approximation to the total cost, the following formula can be adopted :

$$
\mathrm{P}_{i}=\mathrm{P}\left[(1-\alpha)+\alpha\left(\frac{v_{n}}{1.5 v_{m}}\right)^{3}\right]
$$

$P_{i}$ being the total cost for an equipment with any rated wind speed $v_{n}$,

$P$ the total cost for an equipment such that $v_{n}=1.5 v_{m}$,

$\alpha$ represents the fraction, of the total charges, proportional to the installed capacity (alternators, transformers, lines, regulating mechanisms, etc.).

Under these conditions, it can be seen that, for a given value of $\alpha$ (i.e., for a given type of construction) the cost price of the annual energy passes through a minimum for a given value of the rated wind speed, i.e., for a given value of the rated power. In this way the installed capacity constituting the economic optimum for a given type of construction is defined. If the value of the rated wind speed, in relation to the annual average wind speed, is defined, the result will be independent of the site. Thus :

$$
\begin{array}{lll}
\alpha=0.3 & v_{n}=1.5 & v_{m} \\
\alpha=0.2 & v_{n}=1.65 & v_{m} \\
\alpha=0.1 & v_{n}=1.8 & v_{m} \\
\alpha=0 & v_{n}=1.9 & v_{m}
\end{array}
$$

For example, a machine of $25 \mathrm{~m}$ diameter, characterized by $\alpha=0.2$ and required to operate in a district where the annual average wind speed is $8 \mathrm{~m} / \mathrm{s}$, ought to have an installed capacity for a rated wind speed $v_{n}=1.65 \times 8=13.2$ $\mathrm{m} / \mathrm{s}$, i.e., $290 \mathrm{~kW}$ on the shaft. Under these conditions it will generate $610,000 \mathrm{kWh}$ per year.

\section{Different possible features in the construction of the machine}

On broad lines, a windmill for generating electricity will be a high-speed machine having a small number of blades and driving the generator through step-up gearing. The progress made in the construction of gearing, on the one hand, and the high price and the difficulties of constructing generators at very low speed, on the other hand, tend towards the elimination of the direct drive of the generator by the rotor, even for large powers. In the circumstances thus defined, several solutions present themselves on the following points :

NUMBER OF BLADES OF THE ROTOR : The choice will be between two and three blades. A construction with more than three blades it not justified, in view of the advantage of high speeds of rotation; with one blade, which has been used for very small machines, balancing difficulties are too great.

Mounting THE BLADES on THE hub : Two types of installation, one with variable-pitch blades and the other with fixed blades, have been studied and put into operation.

Position of THE Rotor : This can be placed either up-wind or down-wind of the tower. Although the first solution seems preferable to avoid the effect of tower shadow, the second has the advantage of requiring a less expensive method for orientation as a result of the diminution of the required orienting forces.

Electric Generators: There are two possible solutions : the asynchronous generator and the synchronous generator with exciter. The robustness of the first machine and the ease 
with which it can be connected to the network are valuable advantages. On the other hand, the need to take the magnetizing current from the network raises problems in the case of a high installed capacity with an asynchronous machine and gives the advantage to the synchronous machine.

Apart from making a choice from among these different solutions, the principal characteristics have to be specified : the power, the height of the tower and the speed of rotation.

The principal problems just mentioned will be examined in greater detail.

\section{Problems raised by the construction of the blades}

The most delicate problem on the mechanical side of the design of an aerogenerator is raised by the construction of the blades. Although the rest of the design is on proved techniques and classic constructions, the blades, by reason of their dimensions and the particular conditions of stress to which they are subjected, call for special studies and new methods. Contrary to what is frequently thought, the difficulty is not one of aerodynamics but of construction and the strength of materials. Classic methods enable propellers to be designed with efficiencies which are good enough to make it scarcely worth while to try to improve them; this subject need not, therefore, be discussed. The method of constructing the blades, on the other hand, may well be studied with the object of effecting improvements which would result in greater safety in operation and greater economy in manufacture.

There is obviously an advantage in using propellers at high specific speed to reduce both the weight of the rotor itself and that of the gearing. The choice of the specific speed of the rotor may be considered as of the highest importance, since, ultimately it governs the number of blades, the moment of inertia of the rotor, the natural frequencies of vibration and even the characteristics of operation. It is therefore with this particular problem that we are concerned.

We shall adopt for the calculation of the blade the Stefaniak method (Windrad grösster Leistungsabgabe-Forschung, Bd 19, H.1).

The following terms are used :

$\mathrm{C}_{a}=$ lift coefficient of the aerofoil sections;

$\mathrm{C}_{w 0}=\operatorname{drag}$ coefficient;

$\iota=$ length of chord;

$u=$ tangential speed;

$v=$ wind speed;

$w=$ relative speed;

$$
\begin{aligned}
\mathrm{R} & =\text { radius; } \\
z & =\text { number of blades; } \\
\mathrm{L} & =\text { length of a blade element; } \\
\mathrm{A} & =\text { lift; } \\
r_{a} & =\text { density of the air; } \\
\mathrm{I} & =\text { moment of inertia of the section; } \\
\sigma & =\text { stress. }
\end{aligned}
$$

For a section of the blade situated at a distance $R$ from the axis, we have :

$$
\mathrm{C}_{a} \cdot l \cdot z=\frac{\mathrm{R}}{u / D} \cdot A
$$

$\Lambda$ being a non-dimensional quantity given by the following table as a function of the ratio $u / v$ :

\begin{tabular}{|c|c|c|c|c|c|c|c|c|}
\hline$u / v=$ & 1 & 2 & 3 & 4 & 5 & 6 & 7 & 8 \\
\hline$\Lambda$ & 3.36 & 2.38 & 1.73 & 1.33 & 1.08 & 0.91 & 0.8 & 0.7 \\
\hline \hline$u / v=$ & 9 & 10 & 11 & 12 & 13 & 14 & 15 \\
\hline$\Lambda$ & 0.62 & 0.56 & 0.50 & 0.46 & 0.42 & 0.39 & 0.37 \\
\hline
\end{tabular}

For a rotor of given diameler and given speed of rotation, we thus have the value of the product $\mathrm{C}_{a} . l . z$ along the blade.

The graph in Fig. 11 indicates, as a function of the ratio $u / v$ (of the tangential speed to the wind speed) and of the distance from the axis, the value of the product $C_{a} . l . z$. This graph, which is of general application, enables the blade sections to be immediately determined along the length of any blade; in fact, all the points corresponding to the same blade are situated on the same segment of the straight line passing through the origin, the extremity of which is defined by the radial length of the blade and by the ratio $u / v$ at the blade tip. Fig. 11 shows, as an example, the segment corresponding to a blade of $9 \mathrm{~m}$ radius having a ratio $u / v$ of 9 at the blade tip.

Suppose that the same blade section is used in the same conditions of incidence and lift-todrag ratio, i.e., the Gottingen 624 aerofoil section (aerofoil section giving the best lift-to-drag ratio for the maximum relative thickness) used at its greatest lift-to-drag ratio, $\mathrm{C}_{w} / \mathrm{C}_{a}=2 / 100$, incidence $3^{\circ}$ and $\mathrm{C}_{a}=0.92$, the length of chord of the blade section considered is then immediately obtained, the number of blades being assumed to 
have been chosen. 'The lift $\mathrm{A}$ of a blade element of length $\mathrm{L}$ is then written as :

$$
\mathrm{A}=\mathrm{C}_{a} \frac{\gamma w^{2}}{2 g} \cdot l \cdot \mathrm{L}
$$

It is thus possible to determine, along the blade envisaged, the distribution of the lift and, consequently, the distribution of the bending moment $\mathbf{M}_{f}$. As the blade sections are known along the whole length of the blade, the moments of inertia of these sections can be calculated and, finally, the stresses.

For the Gottingen 624 aerofoil section considered, having $160 \mathrm{~mm}$ maximum thickness for a chord of $1,000 \mathrm{~mm}$, the longitudinal neutral axis $\mathrm{XX}^{\prime}$ is at $58 \mathrm{~mm}$ from the face side. The
It can easily be seen that, for similar rotors having the same value of $u / v$ and operating in the same wind, the stresses at corresponding points are identical. The results obtained are therefore independent of the dimensions of the rotor. The graph in Fig. 14 represents the stresses obtained as a function of the ratio $u / v$ at the blade tip and of the distance from the axis, the radius of the rotor being taken as unity.

It is seen that the stress increases rapidly when the ratio $u / v$ increases. This, with the reduction of efficiency, limits the possibilities of constructing high-speed rotors. In addition, the maximum stress in the blade does not occur at the root, but at a distance of approximately 0.6 of the length of the blade. For three-bladed rotors, the maximum stresses are the following:

\begin{tabular}{|c|c|c|c|c|c|c|c|c|c|c|c|c|}
\hline & $u / v=$ & 5 & 6 & 7 & 8 & 9 & 10 & 11 & 12 & 13 & 14 & 15 \\
\hline Solid cross section.. & $\begin{array}{c}\sigma_{\max }= \\
\left(\text { in } \mathrm{kg} / \mathrm{cm}^{2}\right)\end{array}$ & 26 & 80 & 200 & 450 & 900 & 1,635 & 2,800 & 4,500 & 7,500 & 12,000 & 16,350 \\
\hline $\begin{array}{l}\text { Hollow cross section } \\
\text { thickness equal to } \\
0.1 \text { of the thickness } \\
\text { of the blade section. }\end{array}$ & $\begin{array}{c}\sigma_{\max x}= \\
\left(\text { in } \mathrm{kg} / \mathrm{cm}^{2}\right)\end{array}$ & 43 & 132 & 330 & 740 & 1,420 & 2,700 & 4,650 & 7,400 & 12,400 & 19,900 & 27,000 \\
\hline
\end{tabular}

moment of inertia about this axis has the following value :

$\mathrm{I}_{x}=20,000 \mathrm{~cm}^{4}$, area $=1,100 \mathrm{~cm}^{2}$ for a solid cross section.

For a hollow cross section of thickness $e$, if $e=4 \mathrm{~cm}, \mathrm{I}_{x: w^{\prime}}=18,400 \mathrm{~cm}^{4}$ and the area $=880 \mathrm{~cm}^{2}$ if $e=2 \mathrm{~cm}, \mathrm{I}_{x x}=13,750 \mathrm{~cm}^{4}$ and the area $=440 \mathrm{~cm}^{2}$ if $e=1 \mathrm{~cm}, \mathrm{I}_{x x}=8,500 \mathrm{~cm}^{4}$ and the area $=220 \mathrm{~cm}^{2}$

(a) CaSE IN Which the aXis OF The blades is PERPENDICULAR TO THE AXIS OF ROTATION OF THE ROTOR.

From these data, it is possible to calculate the stress in the blade in normal operation. These calculations have been made on the following hypotheses :

- Normal operation in a constant and uniformly distributed wind speed of $12 \mathrm{~m} / \mathrm{s}$, no wind gradient,

-... Axis of the blades perpendicular to the axis of rotation,

-.. Centrifugal force neglected,

- Solid blades,

-. Two-bladed and three-bladed rotor.
For two-bladed rotors with the same ratio $u / v$ at the blade tip, the stresses are 0.45 times those of the three-bladed rotors, hence the advantage of two-bladed rotors at high speeds.

The stresses just determined occur in normal operation. Nevertheless, larger forces will be encountered, particularly in changes of direction in high winds; the corresponding increase is, however, less with high values of $u / v$. It can be considered that, for a change in direction of $40^{\circ}$ in a wind of $40 \mathrm{~m} / \mathrm{s}$ and with variablepitch blades, the increase is 1.5 for $u / v=5$, 1.25 for $u / v=10$ and 1.15 for $u / v=15$. It is necessary, in addition to take into account the gyroscopic forces during orientation. Assuming an identical speed of orientation for all the machines, the gyroscopic stress can be taken as equal to 0.4 times the normal stress for $u / v=5$, 0.06 times the normal stress for $u / v=10$ and 0.017 for $u / v=15$. Finally, the maximum stresses in the blade will be deduced from the stresses in normal operation previously calculated by multiplying by a coefficient $\mathrm{X}$ depending on $u / v$. For a three-bladed rotor, the following values apply : 


\begin{tabular}{|c|c|c|c|c|c|c|c|c|c|c|c|c|}
\hline & $u / v=$ & 5 & 6 & 7 & 8 & 9 & 10 & 11 & 12 & 13 & 14 & 15 \\
\hline & $\mathrm{x}$ & 1.9 & 1.71 & 1.56 & 1.46 & 1.38 & 1.31 & 1.26 & 1.23 & 1.20 & 1.18 & 1.167 \\
\hline Solid cross section. & $\begin{array}{c}\sigma_{\max }= \\
\left(\text { in } \mathrm{kg} / \mathrm{cm}^{2}\right)\end{array}$ & 49.5 & 137 & 312 & 655 & 1,240 & 2,130 & 3,520 & 5,500 & 9,000 & 14,200 & 19,000 \\
\hline Hollow cross section & $\begin{array}{c}\sigma_{\max }= \\
\left(\text { in kg } / \mathrm{cm}^{2}\right)\end{array}$ & 82 & 226 & 520 & 1,090 & 2,050 & 3,550 & 5,820 & 9,150 & 14,900 & 23,500 & 31,500 \\
\hline
\end{tabular}

For a two-bladed rotor, these values should be multiplied by 0.45 .

There remains the case in which the rotor is stopped with the blades face side to wind in very strong winds. The blade is then like a flat plate having the same shape as its projection on a plane perpendicular to the axis of the rotor. The calculation is made by adopting a maximum wind speed of $63 \mathrm{~m} / \mathrm{s}$ (a value corresponding, for a height of $20 \mathrm{~m}$ above flat ground, to a wind speed of $55 \mathrm{~m} / \mathrm{s}$ at a height of $10 \mathrm{~m}$ ). As previously for similar rotor's, the stresses at corresponding points are identical. The results of the calculation can therefore be represented as in Fig. 15 giving the stresses as a function of the ratio $u / v$ at the blade tip and of the distance from the axis, the radius of the rotor being taken as unity. The graph is drawn for a three-bladed rotor. For a two-bladed rotor, the values indicated should be multiplied by 0.45 .

Again, it can be seen here that, as a result of the shape of the blade, the maximum stresses do not occur at the root of the blade, but at a distance from the axis approximately 0.6 of the total length. Comparison of the graphs in Figs. 14 and 15 shows that, at low values of $u / v$, the stress at standstill, facing the maximum wind, is greater than that which occurs in normal operation, but, as $u / v$ increases, the latter very rapidly becomes the greater, so that the standstill stress when the machine is facing the maximum wind is not a factor in limiting the permissible values of $u / v$. If, on the one hand, the increase of the stresses in the blade is taken into account and, on the other, the reduction of the efficiency $\left({ }^{*}\right)$, it does not appear possible, with the technical knowledge at present available, to construct blades economically with a ratio $u / v$ exceeding 9 . It even seems that the

(*) The true efficiency of the rotor decreases rapidly as $u / v$ increases. For the blade which has just been studied, the following values can be taken: $u / v=5$, $\eta=85 \% ; u / v=10, \eta=70 \% ; u / v=15, \eta=55 \%$. best value is distinctly lower, lying between 5 and 7 , according to the type of construction adopted.

(b) CASE IN Which the AXIS OF THE bLADES MAKES, WTTH THE AXIS OF THE ROTOR, AN ANGLE OTHER THAN $90^{\circ}$.

This arrangement, generally called " raking", enables, through the combination of the aerodynamic forces with the centrifugal forces, the bending stresses in the blade to be replaced by tensile stresses.

Let $\mathrm{F}_{\sigma}$ be the centrifugal force exerted on the blade element, $\mathrm{F}_{f}$ the aerodynamic bending force; the resultant force $\mathrm{F}_{t}$ will produce tension in the blade if it is directed along the blade, i.e., if :

$$
\tan \hat{z}=\frac{\mathbf{F}_{f}}{\mathbf{F}_{t}}
$$

o being the angle which the blade makes with the plane perpendicular to the axis of rotation.

This combination can, obviously, be obtained only for a certain value of $\mathrm{F}_{f}$, since the centrifugal force, like the speed of rotation, is constant. In the case of fixed blades, sections which stall can be chosen in order to avoid excessive variations of $\mathrm{F}_{f}$ with the wind speed. Nevertheless, the difficulty remains when there are changes in direction because deflections, which may be large, appear.

If the blade is flexible enough, arrangements can be made so that an increase of wind thrust produces an increase of the rake angle such that the resultant of the forces is always along the axis of the blade. This can be achieved either by elastic flexing of the blade, or by deflection from its root like the ribs of an umbrella-a method called "coning". Independent coning for each blade has been used and recommended, but it is far from being without danger as a result of the dynamic disequilibrium, giving rise to vibrations, which it causes in the rotor, pre- 
cisely at a moment of increased stress. The degree of flexibility of the blade used to compensate the bending by the centrifugal forces necessitates a careful measurement of the masses and the elasticity. Note, also, that great flexibility causes a bending moment to occur near the fixing of the blade at the hub. Whatever arrangement is adopted, when the rotor is stationary facing the wind, bending forces reappear as in the case of the blade perpendicular to the axis of rotation.

To return to the calculation of the forces as in the case of the blade perpendicular to the axis of rotation, it is assumed that the shape of the blade and the cross sections are identical, so that the aerodynamic forces are the same. As previously, the stresses due to the centrifugal forces are independent of the dimensions at corresponding points and for similar rotors. Fig. 17 gives the stress in the blade as a function of the ratio $u / v$ at the blade tip and of the distance from the axis, the radial length of the blade being taken as unity. This is in the case of a three-bladed rotor with solid blades and rake angle $\delta=10^{\circ}$. For the small angles considered, the stress is inversely proportional to the angle $\delta$. For a two-bladed rotor, the stress is two-thirds of that of the three-bladed rotor.

The stresses are thus much less than those which occur in the case of a blade working entirely in bending. As a result of the shape of the blade, the maximum stress occurs in the outermost third of the blade and not at the root.

As has been said above, to enable the centrifugal force, by combining with the aerodynamic forces, to suppress the deflections in the blade, it is necessary to choose suitably the distribution of the masses of the blade. The aerodynamic force $F_{f}$ is imposed by the operating conditions of the machine; the corresponding centrifugal force to eliminate the deflection is :

$$
\mathbf{F}_{e}=\frac{\mathbf{F}_{f}}{\tan \delta}
$$

If $\mathrm{V}$ is the volume of a blade element situated at a distance $R$ from axis and moving with a tangential speed $u$, the density $\gamma$ of this blade element, assumed to be the same in the whole section, will be :

$$
\gamma=g \cdot \frac{\mathrm{F}_{c} \cdot \mathrm{R}}{\mathrm{V} \cdot u^{2}}
$$

A calculation has been made of the values of the densities necessary for a three-bladed rotor with $\delta=10^{\circ}$ in the preceding operating conditions. The results of this calculation are given in Fig. 18, which shows the necessity of using low densities. It will often be difficult to obtain directly the desired densities; they can be achieved by the use of materials of high density, comprising a large number of holes to give a fine cellular structure of which the average density corresponds to that required. Under those conditions, it is necessary to consider not the stress $\sigma$, such as it has been calculated previously (Fig. 17), but the ratio $\sigma / \gamma$ representing the stress brought to unit density. It can then be seen that the real stresses are distinctly higher than a simple examination of Fig. 17 would lead one to suppose.

Although the centrifugal force enables, by raking, the bending in normal operation to be eliminated to some extent, this is no longer so when the blade is at standstill facing the wind. It is necessary to examine what the stress is in this case. The forces when the machine is at standstill facing the wind, for the maximum wind speed of $63 \mathrm{~m} / \mathrm{s}$ which was considered, have been calculated in paragraph $(a)$; they are obviously the same here. The stresses for a solid section are also the same but, taking into account the low densities to be used, we must consider not the values of $\sigma$ but the values of $\sigma / \gamma$ which rapidly become very large. Fig. 19 gives, by the method of representation that has been used up to the present, the variations of $\sigma / \gamma$ as a function of the ratio $u / v$ at the blade tip for a three-bladed rotor at standstill facing a wind of $63 \mathrm{~m} / \mathrm{s}$, the blade being made of homogeneous cellular material. Fig. 20 gives analogous results for a hollow blade made of steel, the metal being placed at the periphery of the blade section and the thickness being chosen so that the desired distribution of mass is obtained. The latter case is obviously more favourable because the material is better used to resist bending. Nevertheless, it can be seen that the stresses at standstill facing the wind are much higher than for a blade which does not use the centrifugal effect. In addition, the maximum stress occurs at the root of the blade. The difficulty can be removed by adopting a distribution of mass along the length of the blade such that the compensation is not made at the root of the blade where it is, moreover, of less advantage. Never theless, the forces when the machine is at standstill, facing the wind, will limit the possibilities of construction in the case of the rotor with raked blades.

The following conclusions may be drawn from this examination. The blade perpendicular to the axis of the rotor may reasonably, with ordinary materials, be used up to a maximum tipspeed ratio of the order of 9 , but its economic use appears to be between 6 and 7 . For this type of blade, the stress when the rotor is at standstill is low in relation to the stress in normal operation.

The blade which is raked enables higher 
speeds to be reached, but, economically, its speed will be limited by the reduction of efficiency. The force when the rotor is at standstill, facing the maximum wind, causes the maximum stresses and will limit the permissible tip-speed ratio to about 10 to 11 -a tip-speed ratio which, moreover, would involve excessive reductions of efficiency. The first type of construction is suitable for variable-pitch blades, the second for fixedpitch blades controlled by stalling.

We have seeen, in another connection, the advantage of two-bladed rotors because they are subjected to less stress at the same speed of rotation. But other factors besides the stress must be taken into consideration, particularly the vibrations. When it is rotating, a two-bladed propeller presents a cyclic variation of its moment of inertia about the pintle axis, which passes successively trough a maximum and through zero twice per revolution. This results, during the movements of orientation, in a cyclic variation of the gyroscopic torque, which may give rise to vibrations. Such a phenomenon no longer exists when the number of blades is three or more.

In addition, when a blade is rotating in a wind with a vertical gradient, which is generally the case, it is subjected to aerodynamic forces undergoing a cyclic variations. Consequently there is a cyclic variation of the reactions of the rotor on its supports and, in particular, a torque exists with cyclic variation which has a tendency to make the machine oscillate about its axis of orientation. This torque has two maxima and two minima per revolution of the rotor for a two-bladed propeller, and three maxima and three minima per revolution for a three-bladed propeller. For a given wind and wind gradient, and for a given diameter of rotor and speed, the amplitude of the variation of this torque, about the pintle axis, is ten times greater with the twobladed rotor than with the three-bladed rotor.

Although the two-bladed rotor is more economical in construction and faster than the threebladed rotor, it does, on the other hand, give rise to vibrations and necessitates special precautions. For this reason, it cannot be recommended in a machine which is already, by its nature, subject to vibrations; it is therefore not surprising that most of the installations and projects have threebladed rotors.

\section{Problems raised by the electrical equipment}

We shall not discuss here wind-driven generators working autonomously, generally with a battery of accumulators, but only machines designed to feed into a high-power alternatingcurrent network. Apart from the exigencies of the network, the aerogeneralor produces special conditions of operation which will influence the choice of the electrical equipment. These conditions are : the use of robust and economical equipment recquiring only the minimum of maintenance, entirely automatic operation, frequent and rapid variation of power, very frequent connections to the network, torque undergoing cyclic variations, the whole installation submitted to very severe atmospheric conditions and large vibrations.

Whatever electrical solution is adopted, mechanical regulation of the power is necessary. This regulation can be effected either by orientation of the blades, or by air brake, or it can be purely aerodynamic by the use of blade sections working at the rated power near their stalling point. The limiting value can be either the speed, or the power, or an aerodynamic thrust on a fixed or rotating part. Although regulation by tachometer is suitable for windmills driving direct-current generators, for machines feeding into a network at constant frequency, power regulation is much better. The mechanical regulator of the power of the windmill will most often serve for power limitation; it will also provide speed limitation when the generator is disconnected from the network. It will therefore have to include a wattmeter element and a tachometer element; the latter can be reduced to a simple centrifugal limiting device. For a fixed-bladed machine using sections which stall, the rotor will have to have a braking system to avoid racing if the machine is disconnected from the network. The necessity for this braking system, which is always of doubtful efficacy, is a serious disadvantage of the fixed-bladed machine. The best method, for fixed blades, consists not in absorbing the torque by a brake on the shaft, but by an aerodynamic system at the end of the blades, which is much safer in operation.

Several solutions may be adopted for the production of alternating current and constant frequency. These fall into three categories:

(1) An intermediate set between the windmill and a standard synchronous generator coupled to the network;

(2) An asynchronous generator driven directly by the windmill and coupled to the network;

(3) A synchronous generator driven directly by the windmill and coupled to the network.

In the first category there are various methods of which the following are the most interesting:

(a) A direct-current generator driven by the windmill and not needing very precise regulation of the speed of rotation. The direct current thus 
generated is then transformed into alternating current, either by an inverter or by a rotary converter. The conversion can be made either for each aerogenerator or for a group of aerogenerators. Recent advances in the development of electrostatic generators do not exclude the possibility of generating direct current at high voltage. The power can be transported some distance and then transformed, if necessary, for the needs of utilization.

(b) A standard synchronous generator, coupled to the network and driven by an air turbine which is itself supplied by the depression created by an aerogenerator with hollow blades (Andreau method). It is curious that the large $100-\mathrm{kW}$ machine designed on this principle does not use the principal advantage that this arrangement offers, i.e., the possibility of transferring the regulation to the air turbine, of small dimensions, and of using a rotor with fixed blades. On the contrary, a rotor with variable-pitch blades has been adopted, causing considerable mechanical complication. Under these conditions, the sole peculiarities of the machine are its indisputable originality and also its low efficiency.

(c) A better method would be to use, as an intermediary between the propeller and the generator, hydraulic transmission effected by a gear pump delivering into a common pipe connecting several windmills and feeding a turbine driving the standard alternator. It would be an advantage in this case to install the pump in place of the gearing. The efficiency of such a transmission might be of the order of $80 \%$ and therefore much higher than that of the air transmission of the hollow-bladed windmill. The use of several windmills to supply the same generator would have the advantage of giving a more regular production of energy necessitating less frequent connections to the network. In addition, if a turbine of the Pelton type were used (which is advisable, for such a transmission must be at high pressure to remain economic), there would be no disadvantage in leaving the alternator coupled permanently to the network, the rotor of the turbine turning in the air if production from the windmills stopped.

The asynchronous generator seems to provide the most economic and robust solution. Apart from its simplicity of construction and the absence of rotating contacts, it has, when driven by a windmill, two great advantages: ease of connection to the network and the absence of the risk of oscillation. There is nothing to prevent the machine being switched in with a speed different, by several per cent, from the synchronous speed, the resulting overload being of very short duration. The speed at switchingin may be found by means of a simple tacho- meter. Apart from the usual protection, the switchgear then consists only of a tachometer contact controlling the switching-in to the network and a reverse current relay ensuring disconnection if the wind drops. Although the asynchronous generator does not need an exciter, it takes its magnetizing current from the network and absorbs reactive power. This peculiarity is not a disadvantage if the power supplied by the windmill is low in relation to that of the network; it raises a problem as soon as the power supplied by the group of asynchronous generators becomes large. The use of batteries of static capacitors enables the power factor to be improved. Finally, rotating sets, suitably distributed on the line fed by asynchronous aerogenerators, can be called upon to supply the necessary magnetizing current.

When the wind power available is low in relation to the total power of the network, it is possible to use a generator with a resistant cage and large slip. This generator is driven by a windmill having its power regulated simply by orientation of the blades and equilibration of the forces on the blades by a spring. This method of regulation, which is very robust and needs no servo-motor, is characterized by a certain difference of speed which, taking into account the steep slope of the torque characteristic of the large-slip generator, is represented by only a slight relative difference of power. In this way it would be possible to construct a machine having its power limited without either servo-motor or governor.

The synchronous generator has the great advantage of itself supplying, by its exciter, a magnetizing current. This seems to favour its use for large powers. It has, however, in its standard form, some disadvantages. The exciter, being a commutator machine, tends to increase the cost of the set and necessitates more supervision than is required by the asvnchronous machine, which has no rotating contacts. It is perhaps possible to overcome this difficulty by the use of self-excited alternators, the excitation being supplied by a special winding on the stator and a rectifier.

While, in the asynchronous senerator, with variations in slip, the electric power fed into the network corresponds attomatically with the mechanical power available on the shaft, in the synchronous generator. on the other hand, it is necessary to adjust the value of the exciting current in proportion to the nower to be exchanged. hence the need for electrical reau. lation of the excitation if the power factor is to be maintained at a suitable value. This electrical regulation is added to the mechanical resnlation of the nower of the windmill. Nevertheless, advantage can be taken of the charac- 
teristics of synchronous machines in a group of aerogenerators, some equipped with synchronous generators and others with asynchronous generators. In such a combination, the magnetizing current of the asynchronous generators would be provided by the synchronous generators, suitably over-excited.

The connection of the synchronous generator to the network, which demands a much more precise adjustment of the speed of rotation than the connection of the asynchronous machine, raises a serious problem, as this operation has to be performed frequently for aerogenerators. It was thought that a solution would be provided by interposing a free-running coupling between the windmill and the generator and leaving the later coupled permanently to the network. But this merely shifts the difficulty, for such couplings, for high powers, are very uneconomic.

A more interesting solution consists in equipping the rotor of the generator with large dampers permitting asynchronous operation at the time of switching-in. Such dampers are also desirable on account of the risk of oscillation with synchronous generators. These machines, in fact, possess a natural period of oscillation depending on their moment of inertia and on the synchronizing torque.

$$
\mathrm{T}=\frac{0.25}{p} \sqrt{\frac{\mathrm{PD}^{2} \cdot f}{\left(\mathrm{I}_{k} / \mathrm{I}\right) \mathrm{N}}}
$$

$\mathrm{T}$ being the period (in seconds), $p$ the number of pairs of poles, $f$ the frequency, $\mathrm{PD}^{2}$ being in $\mathrm{kg} \cdot \mathrm{m}^{2}, \mathrm{I}_{k} / \mathrm{I}$ the short-circuit ratio, and $\mathrm{N}$ the kVA.

The form of this expression for the natural period of oscillation shows that it depends on the state of load of the machine; it varies therefore over a certain range. This is a cause of difficulty because the period of oscillation of the generator, or its harmonics, must be prevented from coinciding with the period of the forces producing oscillation of the windmill (passage of the blades in front of the tower, etc.).

This brief examination shows that, although a windmill of high installed capacity necessitates the use, perhaps partial, of synchronous generators, these will be, by reason of their particular conditions of utilization, of a somewhat different design from the standard generators. The choice and proportion of synchronous and asynchronous machines will depend on a study of the network.

(To be continued.)

\section{NOTRE FRONTISPICE}

ADAM DE CRAPPONNE (1526-1576).

Détourner le lit d'une rivière ou obliger ses eaux à changer de bassin versant sont choses courantes aujourd'hui. Au XVI siècle, c'était un dessein hardi et revolutionnaire. C'est pourtant $c$ qu'osa faire Adam de Crapponne avec la Durance pour irrigues lat Crau on avec l'Argens et le Reyran pour désensabler le port đe Fréjus. Et, anx yetx de qui se penche sur le récent amé nagement de la Durance à Serre-Poncon, ce demier apparait bien conme calqué sur les conceptions d'Adam de Crapponne et comme une sorte de prolongement de son euvre.

Adam de Crapponne, né à Sulon-de-Provence en 1526, fut incontestablement le précurseur de Riquet et de Vauban en matière de canaux et de fortifications. Outre le canal provençal qui porte son nom, on lui doit des projets ét des études sur plusieurs canaux en France et strt le dessechement de marais; également les fortifications de Metz et de Nice.

Son personnage s'affre $\dot{a}$ nous conme celui du premier en tate parmi les grands ingénieurs hydraticiens on militaires de France. Son atvre, accomplie dans une épogtte fort tourmentée, est marquée par une grande hardiesse de vues, par l'énergie déployée contre des obstacles sans nombre, le sacrifice de sa fortune et son attitude stoique devant les insuccès, les mo-

I1 mourtut, empoisonné, dit-on, à Nantes, en 1576 .
ADAM DE CRAPPONNE (1526-1576).

It is quite a common thing nowadays to divert a river or to make it flow into a different watershed. In the XVI century it wats daring and revolutionary to andertake such frojects Nevertheless, that is what Adan de Crapponnc did to the Durance in order to irrigate the Cran, and ho used the Argens and the Reyran in a similar way to desilt Frejus harbowr. Those acquainted with the Serre-Poncon project on the Durance will see that it seens to be based on Adam de Crapponne's ideas and that it has cvery appearance of being a sort of cotension of his work.

Born at Salon-de.Prowence in 1526. Adam de Crapponne was indubitably a forcrumer of Riquet and Vauban as far as canals and fortifications are conconed. In addition to the Provencal cantal named after him, he wits responsible for enginecring various French canals and marsh reclamation projects and he designed the fortifications at . Netz and Nice.

He was the first great French hydrallics and military engineer. His accomplishments, products of an crtremely troubled age are characterised by a very bold autlook, by energetic action in the face of countess abstacles, by the sacmite of his fortune and by his stoicism in the face of failure, mockery, insults, and

It is said that he dicd of poisoning at Nantes in 1576. 